\title{
MEASURES WITH BOUNDED CONVOLUTION POWERS
}

\author{
BY \\ BERTRAM M. SCHREIBER( $\left.{ }^{1}\right)$
}

\begin{abstract}
For an element $x$ in a Banach algebra we study the condition

$$
\sup _{n \geqq 1}\left\|x^{n}\right\|<\infty .
$$
\end{abstract}

Although our main results are obtained for the algebras $M(G)$ of finite complex measures on a locally compact abelian group, we begin by considering the question of bounded powers from the point of view of general Banach-algebra theory. We collect some results relating to (1) for an element whose spectrum lies in the unit disc $D$ and has only isolated points on $\partial D$. There follows a localization theorem for commutative, regular, semisimple algebras $A$ which says that whether or not (1) is satisfied for an element $x \in A$ with spectral radius 1 is determined by the behavior of its Gelfand transform $\hat{x}$ on any neighborhood of the points where $|\hat{x}|=1$. We conclude the general theory with remarks on the growth rates of powers of elements not satisfying (1).

After some applications of earlier results to the algebras $M(G)$, we prove our main theorem. Namely, we obtain strong necessary conditions on the Fourier transform for a measure to satisfy (1). Some consequences of this theorem and related results follow. Via the generalization of a result of G. Strang, sufficient conditions for (1) to hold are obtained for functions in $L^{1}(G)$ satisfying certain differentiability conditions. We conclude with the result that, for a certain class $\mathscr{G}$ of locally compact groups containing all abelian and all compact groups, a group $G \in \mathscr{G}$ has the property that every function in $L^{1}(G)$ with spectral radius one satisfies (1) if and only if $G$ is compact and abelian.

1. Introduction. Conditions similar to (1) and consequences of it appear in a number of contexts in analysis. For example, it is known [32], [8, pp. 221-222] that if $X$ is a Banach [Hilbert] space and $G$ is a uniformly bounded, commutative group of operators on $X$, then there exists an equivalent [Hilbert] norm on $X$

Presented to the Society, January 24, 1968; received by the editors September 11, 1969.

AMS 1969 Subject Classifications. Primary 4250, 4256, 4655, 4680; Secondary 2265, 4225, 4240, 4251, 4252, 4650, 4730, 4745, 4750.

Key Words and Phrases. Bounded iterates, convolution powers, singly-generated algebras, spectral projection, semisimple algebra, regular algebra, partition of unity, localization, extreme point, growth rate, Bohr compactification, idempotent measure, coset ring, homomorphism of group algebras, piecewise affine map, compactly generated group, extension of FourierStieltjes transforms, differentiable Fourier transforms, commutator subgroup.

(1) This paper constitutes part of the author's doctoral dissertation [27]. He would like to express his appreciation to Professor Irving Glicksberg for his kind encouragement and many helpful suggestions during the preparation of that thesis, and to the National Science Foundation and the University of Washington for their generous financial support. 
under which every element of $G$ is an isometry. (1) and its consequence, the boundedness of the sequence $y_{n}=n^{-1} \sum_{0}^{n} x^{k}$, form one of the bases of ergodic theory (see $[9, \mathrm{pp} .660 \mathrm{ff}])$. In the analytical theory of semigroups it is shown, via the Hille-Yosida-Phillips Theorem [9, p. 624], that the infinitesimal generators of semigroups of operators give rise to families of operators, parameterized by a real parameter $\lambda$, which satisfy (1) uniformly in $\lambda$. Finally, we mention the stability of linear, finite-difference approximations to the solution of an initial-value problem in partial differential equations; we refer the reader to [23] for details.

(1.1) Notation. Given a Banach algebra $A$ and $x \in A$ we shall denote the spectrum of $x$ in $A$ by $\mathrm{sp}_{A}(x)$, the spectral radius of $x$ by $r(x)$, the resolvent of $x$ at $\lambda \notin \operatorname{sp}_{A}(x)$ by $R(\lambda, x)$, and the closed subalgebra of $A$ generated by $x$ (and the identity of $A$ if it exists) as $A(x)$. If $A$ is commutative, $\mathscr{M}_{A}$ will denote its maximal ideal space. Let $A_{e}=A$ if $A$ has an identity, and if not let $A_{e}$ be the algebra $A$ with the identity adjoined. All algebras will be assumed complex. If $X$ is a Banach space, $B(X)$ is the Banach algebra of bounded operators on $X$. Let the set of elements in $A$ which satisfy (1) be $\mathscr{B}(A)$.

If $f$ is a bounded complex-valued function on a set $X$ and $E \subset X$, let $\|f\|_{X}$ $=\sup _{x \in X}|f(x)|, E^{\prime}=X \backslash E$, and $\chi_{E}$ be the characteristic function of the set $E$. If $X$ is a locally compact Hausdorff space, denote by $C(X), C_{0}(X)$, and $C_{00}(X)$ the space of bounded complex-valued continuous functions on $X$, the space of functions in $C(X)$ which vanish at infinity, and the continuous functions with compact support in $X$, respectively.

For further standard notation and results on operator theory and the general theory of Banach algebras we shall follow [9], [17], [21], or [24].

(1.2) THEOREM. Let $A$ be a Banach algebra.

(i) $\mathscr{B}(A)$ is a balanced set. If $x, y \in \mathscr{B}(A)$ and $x y=y x$, and if $0<\alpha<1$, then $\alpha x+(1-\alpha) y \in \mathscr{B}(A)$. Thus if $A$ is commutative $\mathscr{B}(A)$ is convex.

(ii) If $x, y \in \mathscr{B}(A)$ and $x y=y x$, then $x y \in \mathscr{B}(A)$. Hence $\mathscr{B}(A)$ is closed under multiplication if $A$ is commutative.

(iii) If $x^{n} \in \mathscr{B}(A)$ for some positive integer $n$, then $x \in \mathscr{B}(A)$.

(iv) Every element of $\mathscr{B}(A)$ has spectral radius at most one.

(v) If $x$ has norm $\leqq 1$ or spectral radius $<1$, then $x \in \mathscr{B}(A)$.

(vi) $\mathscr{B}(A)$ is contained in the closure of its interior.

(vii) $\mathscr{B}(A)$ is closed if and only if it contains every member of $A$ with spectral radius 1 , and it is open if and only if it contains no elements of spectral radius 1.

Proof. $\mathscr{B}(A)$ is clearly balanced. Let $x, y \in \mathscr{B}(A)$, say $\left\|x^{n}\right\| \leqq M,\left\|y^{n}\right\| \leqq N$, $n=1,2, \ldots$, with $x y=y x$, and let $0<\alpha<1$. Then

$$
\begin{aligned}
\|\left(\alpha x+(1-\alpha) y^{n} \|\right. & \leqq \alpha^{n} M+\sum_{k=1}^{n-1}\left(\begin{array}{l}
n \\
k
\end{array}\right) \alpha^{n-k}(1-\alpha)^{k} M N+(1-\alpha)^{n} N \\
& \leqq M N \sum_{k=0}^{n}\left(\begin{array}{l}
n \\
k
\end{array}\right) \alpha^{n-k}(1-\alpha)^{k}=M N
\end{aligned}
$$


This says $\alpha x+(1-\alpha) y \in \mathscr{B}(A)$, and (i) is proven.

(ii) is obvious, as are (iv) and (v). Suppose that $x^{n} \in \mathscr{B}(A)$, say $\left\|x^{n k}\right\| \leqq M$, $k=1,2, \ldots$, and let $N=\max _{1 \leqq p<n}\left\|x^{p}\right\|$. Then for $m=1,2, \ldots$ we have $m=n k+p$ with $0 \leqq p<n$, so $\left\|x^{m}\right\|=\left\|x^{n k+p}\right\| \leqq\left\|x^{n k}\right\|\left\|x^{p}\right\| \leqq M N$, giving (iii).

Let $x \in \mathscr{B}(A)$. If $x$ has spectral radius less than one, then by the continuity of the spectrum [24, Theorem 1.6.16, pp. 35-36] there is a neighborhood of $x$ all of whose elements have spectral radius less than one and are thus in $\mathscr{B}(A)$ by $(\mathrm{v})$. For $x$ with spectral radius 1 and $\varepsilon>0,(1-\varepsilon) x$ has spectral radius $(1-\varepsilon)<1$ and hence is in the interior of $\mathscr{B}(A)$. (vi) now follows from (iv). We have shown, in fact, that the closure of the interior of $\mathscr{B}(A)$ consists of all elements of $A$ with spectral radius at most one, and this gives the first half of (vii). The other half follows, again, from the continuity of the spectrum, (iv) and (v).

REMARK. There are, of course, many algebras $A$ in which $\mathscr{B}(A)$ is closed. $\mathscr{B}(A)$ is open if $A$ is a radical algebra; some less trivial examples where $\mathscr{B}(A)$ is open are given in $\$ 4$.

(1.3) Let $A_{1}$ be the algebra of all functions $f(z)=\sum_{n=1}^{\infty} a_{n} z^{n}$ analytic on the interior of the closed unit disc $D$ in the complex plane and satisfying $\|f\|_{1}=$ $\sum_{n=1}^{\infty}\left|a_{n}\right|<\infty$ (whence $f$ is a continuous function on $D$ ). $A_{1}$ is a Banach algebra under this norm, and $\mathscr{M}_{A_{1}} \cup\{0\}$ is identified with $D$ by point-evaluations. We now note that $A_{1}$ "operates" on $\mathscr{B}(A)$.

Proposition. Let $A$ be a commutative Banach algebra and $x \in \mathscr{B}(A)$. Then there is a unique, bounded algebra homomorphism $H: A_{1} \rightarrow A$ such that

(i) $(H f)^{\wedge}=f \circ \hat{x}, f \in A_{1}$, i.e., $\hat{x}$ is the adjoint of $H$.

(ii) $\|H\|=m(x)=\sup _{n \geqq 1}\left\|x^{n}\right\|$.

Every bounded homomorphism from $A_{1}$ to $A$ satisfies (i) and (ii) for some $x \in \mathscr{B}(A)$.

Proof. If $x \in \mathscr{B}(A)$, let $H$ be defined as follows

$$
H f=\sum_{n=1}^{\infty} a_{n} x^{n}, \quad \text { if } f(z)=\sum_{n=1}^{\infty} a_{n} z^{n} \in A_{1} .
$$

Then $\|H f\| \leqq \sum_{n=1}^{\infty}\left|a_{n}\right|\left\|x^{n}\right\| \leqq m(x)\|f\|_{1}$, so the series (2) is absolutely convergent in $A$, and $H$ is a well-defined algebra homomorphism with $\|H\| \leqq m(x)$. Since $H z^{n}=x^{n}$ and $\left\|z^{n}\right\|_{1}=1, n=1,2, \ldots$, we have $\|H\|=m(x)$. For $M \in \mathscr{M}_{A}, \hat{x}(M) \in D$ by (1.2.iv), and

$$
(H f)^{\wedge}(M)=\sum_{n=1}^{\infty} a_{n} \hat{x}(M)^{n}=f(\hat{x}(M)), \quad f \in A_{1} .
$$

$H$ is unique because it is determined by $H z=x$. And if $H: A_{1} \rightarrow A$ is any homomorphism and $x=H z$, then $\left\|x^{n}\right\| \leqq\|H\|, n=1,2, \ldots$, and $H$ satisfies (2) for this $x$.

2. Some spectral conditions related to $\mathscr{B}(A)$. Our goal in this section is to prove, in a somewhat more general form, that a compact linear operator $T$ on a 
Banach space $X$ is in $\mathscr{B}(B(X))$ if and only if $r(T) \leqq 1$ and each eigenvalue of $T$ of unit modulus is simple $\left({ }^{2}\right)$. The following is a well-known corollary of $[24$, Theorem 1.6 .12$, p. 33]. We let $E^{i s}$ denote the set of isolated points of a subset $E$ of the complex plane, and $\partial E$ denotes the boundary of $E$.

(2.1) LemMA. Let $B$ be a closed subalgebra of the Banach algebra $A$. Then for $x \in B$,

(i) $\operatorname{sp}_{A}(x) \cap\{z:|z|=r(x)\}=\operatorname{sp}_{B}(x) \cap\{z:|z|=r(x)\}$.

(ii) $\left[\operatorname{sp}_{B}(x)\right]^{i s}=\left[\operatorname{sp}_{A}(x)\right]^{i s} \cap \partial \operatorname{sp}_{B}(x)$.

(2.2) Lemma. Let $A$ be a Banach algebra and $x \in A$. Suppose that

(i) $r(x) \leqq 1$,

(ii) $\mathrm{sp}_{A}(x) \cap \partial D$ is discrete in $\mathrm{sp}_{A}(x)$,

(iii) $A\left(x e_{\partial}\right)$ is a semisimple algebra, where $e_{\partial}$ is the spectral projection corresponding to the spectral set $\operatorname{sp}_{A}(x) \cap \partial D$. Then $x \in \mathscr{B}(A)$.

Proof. We may assume that $A$ has an identity $e$. By (2.1), (ii) is satisfied with $\operatorname{sp}_{A}(x)$ replaced by $\operatorname{sp}_{A(x)}(x)$. We may thus assume that $A=A(x)$, which is, of course, commutative.

If $r(x)<1$, then $x \in \mathscr{B}(A)$ by (1.2.v). If $r(x)=1$, let $\mathrm{sp}_{A}(x) \cap \partial D=\left\{\lambda_{1}, \ldots, \lambda_{n}\right\}$ and let $F=\operatorname{sp}_{A}(x) \mid\left\{\lambda_{1}, \ldots, \lambda_{n}\right\}$. Let $e_{i}$ be the spectral projection corresponding to the point $\lambda_{i}, i=1, \ldots, n$, and $e_{0}$ the one corresponding to $F$. Then $e_{1}+\cdots$ $+e_{n}=e_{\partial}, e_{\partial}+e_{0}=e$, and $e_{i} e_{j}=\delta_{j}^{i} e_{i}, i, j=0, \ldots, n$. By the semisimplicity of the ideal $A\left(x e_{\partial}\right)=A(x) e_{\partial}$ in $A$, we have $x e_{i}=\lambda_{i} e_{i}, i=1, \ldots, n$. And $r\left(x e_{0}\right)=\sup _{\lambda \in F}|\lambda|<1$, so that $x e_{0} \in \mathscr{B}(A)$. Thus

$$
\begin{aligned}
\left\|x^{k}\right\| & =\left\|\left(\sum_{i=0}^{n} x e_{i}\right)^{k}\right\|=\left\|\sum_{i=0}^{n}\left(x e_{i}\right)^{k}\right\|=\left\|\left(x e_{0}\right)^{k}+\sum_{i=1}^{n} \lambda_{i}^{k} e_{i}\right\| \\
& \leqq\left\|\left(x e_{0}\right)^{k}\right\|+\sum_{i=1}^{n}\left|\lambda_{i}\right|^{k}\left\|e_{i}\right\|=\left\|\left(x e_{0}\right)^{k}\right\|+\sum_{i=1}^{n}\left\|e_{i}\right\|, \quad k=1,2, \ldots .
\end{aligned}
$$

(2.3) Corollary. Let $A$ be a semisimple commutative Banach algebra with discrete maximal ideal space. Then for $x \in A, x \in \mathscr{B}(A)$ if and only if $r(x) \leqq 1$.

Proof. This follows immediately from Lemma (2.2) and the observation that since $\hat{x} \in C_{0}\left(\mathscr{M}_{A}\right)$, (i) implies (ii) in this case.

(2.4) THEOREM. Let $A$ be a Banach algebra, let $x \in A$ satisfying (2.2.i) and (2.2.ii), and suppose that each point of $\mathrm{sp}_{A}(x) \cap \partial D$ is a pole of $R(\lambda, x)$. Then using the notation established in (2.2), the following are equivalent:

(iii) $A\left(x e_{\partial}\right)$ is semisimple.

(iv) $x e_{i}=\lambda_{i} e_{i}, i=1, \ldots, n$.

(v) $R(\lambda, x)$ has a simple pole at $\lambda_{i}, i=1, \ldots, n$.

${ }^{(2)}$ This result was obtained by Bonsall and Tomiuk [3] using the theory of locally compact semialgebras. See also [19], [20], [22]. 
(vi) For some $C>0,\|R(\lambda, x)\| \leqq C(|\lambda|-1)^{-1},|\lambda|>1$.

(vii) $x \in \mathscr{B}(A)$.

Moreover, if $X$ is a Banach space and $x=T \in B(X)$, then the conditions above imply that

(viii) $\lambda_{i}$ has index 1 , so it is a simple eigenvalue of $T, i=1, \ldots, n$.

Proof. Again assume $e \in A$. Note that if the $e_{i}$ are computed in $A_{e}$ we in fact have $e_{i} \in A, i=1, \ldots, n$.

(iii) implies (vii) by (2.2). Suppose (vii) holds, and let $C=\sup _{n \geqq 1}\left\|x^{n}\right\|$. Recall that for $|\lambda|>\|x\|$ we have

$$
R(\lambda, x)=\sum_{n=0}^{\infty} \frac{x^{n}}{\lambda^{n+1}}
$$

and hence

$$
\begin{aligned}
\|R(\lambda, x)\| & \leqq \sum_{n=0}^{\infty} \frac{\left\|x^{n}\right\|}{|\lambda|^{n+1}} \\
& \leqq C \sum_{n=0}^{\infty} \frac{1}{|\lambda|^{n+1}}=C(|\lambda|-1)^{-1}, \quad|\lambda|>\|x\| .
\end{aligned}
$$

Since the series (3) is thus absolutely convergent for $|\lambda|>1$, it represents the resolvent outside $D$, and (vi) follows.

Fix $i \leqq n$ and expand the resolvent about $\lambda_{i}$ as a Laurent series absolutely convergent in some deleted neighborhood $U$ of $\lambda_{i}$ :

$$
R(\lambda, x)=\sum_{k=-m}^{\infty} a_{k}\left(\lambda-\lambda_{i}\right)^{k}, \quad \lambda \in U,
$$

where $a_{k} \in A,-m \leqq k<\infty$, and $a_{-m} \neq 0$. Assuming that (vi) holds, we have $\left\|\left(\lambda-\lambda_{i}\right) R(\lambda, x)\right\| \leqq C$ if $|\lambda|>1$ and $\arg \lambda=\arg \lambda_{i}$. Thus $m=1$.

The equivalence of (iv) and (v) and the implication (v) $\Rightarrow$ (viii) follow from [9, Theorem VII.3.18, p. 573], which may be put in a general Banach-algebra setting by considering the left regular representation. Finally, if (iv) holds then

$$
A\left(x e_{\partial}\right)=\oplus \sum_{1}^{n} A\left(x e_{i}\right)=\oplus \sum_{1}^{n} A(x) e_{i}=C^{n},
$$

which is certainly semisimple, and the proof is complete.

(2.5) Corollary. Let $T \in B(X)$ satisfying (2.2.i) and (2.2.ii). If $\left(\lambda_{i} I-T\right) E_{i}$ is nilpotent, $i=1, \ldots, n$ (where $E_{i}$ is the spectral projection corresponding to $\lambda_{i}$ ), then $T \in \mathscr{B}(B(X))$ if and only if each $\lambda_{\mathfrak{i}}$ is a simple eigenvalue of $T$. In particular, if $E_{\mathfrak{i}} X$ is finite-dimensional, $i=1, \ldots, n$, then the equivalence holds.

(2.6) Corollary. Let $X$ be a Banach space and T a compact linear operator on $X$. Then $T \in \mathscr{B}(B(X))$ if and only if $r(T) \leqq 1$ and $\lambda$ is a simple eigenvalue of $T$ for every $\lambda \in \operatorname{sp}(T)$ such that $|\lambda|=1$. 
3. The localization theorem. We indicate here how partitions of unity may be utilized to generate elements with bounded powers and prove the localization theorem. As an application of this theorem we can find the extreme points of $\mathscr{B}(A)$ for some algebras $A$.

If $A$ is a Banach algebra and $x_{1}, \ldots, x_{n} \in A$, let $\left[x_{1}, \ldots, x_{n}\right]$ denote the closed ideal in $A$ generated by $x_{1}, \ldots, x_{n}$.

(3.1) Definition. Let $A$ be a commutative Banach algebra and $I$ a closed ideal in $A$. Let $\left\{U_{1}, \ldots, U_{n}\right\}$ be an open covering of $\mathscr{M}_{I}=\mathscr{M}_{A}$ |hull $(I)$. We shall say that $\hat{A}$ contains a partition of unity subordinate to $\left\{U_{1}, \ldots, U_{n}\right\}$ if we can find $\alpha_{1}, \ldots, \alpha_{n} \in A$ such that $\hat{\alpha}_{i} \equiv 0$ on $\mathscr{M}_{I} \mid U_{i}, i=1, \ldots, n$, and $\sum_{i=1}^{n} \hat{\alpha}_{i} \equiv 1$ on $\mathscr{M}_{I}$. Recall that if $A$ is regular and $\left\{U_{0}, U_{1}, \ldots, U_{n}\right\}$ is an open covering of $\mathscr{M}_{A}$ with $U_{0}^{\prime}$ compact, then $\hat{A}_{e}$ contains a partition of unity subordinate to $\left\{U_{0}, \ldots, U_{n}\right\}$ such that $\alpha_{1}, \ldots, \alpha_{n} \in A$.

(3.2) THEOREM. Let I be a closed ideal in the commutative Banach algebra $A$ such that $I$, as an algebra in its own right, is semisimple (i.e., $I \cap$ radical $(A)=\{0\})$. Let $\hat{A}$ contain a partition of unity $\alpha_{1}, \ldots, \alpha_{n}$ subordinate to the open covering $\left\{U_{1}, \ldots\right.$, $\left.U_{n}\right\}$ of $\mathscr{M}_{I}$. Suppose $x_{1}, \ldots, x_{n} \in \mathscr{B}(A)$ such that $\hat{x}_{i} \equiv \hat{x}_{j}$ on $U_{i} \cap U_{j}$ and either $\alpha_{i}$ or $x_{i}$ is in $I$, all $i, j$. Then there is a unique element $x \in I \cap\left[x_{1}, \ldots, x_{n}\right]$ such that $x \in \mathscr{B}(A)$ and $\hat{x} \equiv \hat{x}_{i}$ on $U_{i}, i=1,2, \ldots, n$.

Proof. Define a function $f$ on $\mathscr{M}_{I}$ by $f(M)=\hat{x}_{i}(M)$ if $M \in U_{i}, i=1, \ldots, n$. If $M \in U_{i}$ and $m$ is a positive integer, then

$$
\begin{aligned}
f(M)^{m}=\hat{x}_{i}(M)^{m} & =\left(\sum_{j=1}^{n} \hat{\alpha}_{j}(M)\right) \hat{x}_{i}(M)^{m}=\sum_{\left\{j: M \in U_{j}\right\}} \hat{\alpha}_{j}(M) \hat{x}_{j}(M)^{m} \\
& =\sum_{j=1}^{n} \hat{\alpha}_{j}(M) \hat{x}_{j}(M)^{m} .
\end{aligned}
$$

Thus if we set $x=\sum_{1}^{n} \alpha_{i} x_{i}$ then $x \in I \cap\left[x_{1}, \ldots, x_{n}\right]$ and from the semisimplicity of $I$ we see that $x$ is the unique element of $I$ such that $\hat{x}=f$, and that $x^{m}=\sum \alpha_{i} x_{i}^{m}$, $m=1,2, \ldots$ Thus

$$
\left\|x^{m}\right\|=\left\|\sum_{i=1}^{n} \alpha_{i} x_{i}^{m}\right\| \leqq \sum_{i=1}^{n}\left\|\alpha_{i}\right\|\left\|x_{i}^{m}\right\|,
$$

which is bounded in $m$.

(3.3) LocAlization TheOREM. Let I be a closed ideal in the commutative Banach algebra $A$ such that $I$, as an algebra, is regular and semisimple. Let $x \in I$ with $r(x) \leqq 1$, and let

$$
E_{x}=\left\{M \in \mathscr{M}_{I}:|\hat{x}(M)|=1\right\} .
$$

Suppose that for each $M \in E_{x}$ there exists $x_{M} \in \mathscr{B}(A)$ such that $\hat{x}_{M}=\hat{x}$ on a neighborhood of $M$. Then $x \in \mathscr{B}(A)$.

Proof. Since $E_{x}$ is compact we can find $x_{1}, \ldots, x_{n} \in A$ and open subsets $U_{1}, \ldots$, $U_{n}$ of $\mathscr{M}_{1}$ such that $x_{i} \in \mathscr{B}(A), \hat{x}_{i} \equiv \hat{x}$ on $U_{i}, i=1, \ldots, n$, and $E_{x} \subset U_{1} \cup \cdots \cup U_{n}$. 
For each $\varepsilon>0$, let $E_{x}(\varepsilon)=\{M:|\hat{x}(M)| \geqq 1-\varepsilon\}$, and let $U=U_{1} \cup \cdots \cup U_{n}$. Then since $\hat{x} \in C_{0}\left(\mathscr{M}_{I}\right)$ we have $E_{x}(\varepsilon) \subset U$ for some $\varepsilon>0$. Since $E_{x}(\varepsilon)$ is compact we can choose an open set $U_{0}$ with $E_{x}(\varepsilon) \cap \bar{U}_{0}=\varnothing, U_{0} \cup U=\mathscr{M}_{I}$, and $\mathscr{M}_{I} \mid U_{0}$ compact. By the regularity of $I$ choose $k \in I$ so that

$$
\begin{aligned}
\hat{k}(M) & =1 & & \text { if } M \in E_{x}(\varepsilon) \\
& =0 & & \text { if } M \in \bar{U}_{0} .
\end{aligned}
$$

Let $m$ be so large that $(1-\varepsilon)^{m}\left(1+\|\hat{k}\|_{\mathscr{M}_{I}}\right)<1$, and set $x_{0}=x^{m}-k x^{m} \in I$. If $M \in E_{x}(\varepsilon)$ then $\hat{x}_{0}(M)=\hat{x}(M)^{m}(1-\hat{k}(M))=0$; and if $M \notin E_{x}(\varepsilon)$, then

$$
\left|\hat{x}_{0}(M)\right|=\left|\hat{x}(M)^{m}(1-\hat{k}(M))\right| \leqq|\hat{x}(M)|^{m}(1+|\hat{k}(M)|) \leqq(1-\varepsilon)^{m}\left(1+\|\hat{k}\|_{\mathscr{M}_{1}}\right)<1 .
$$

Thus $\hat{x}_{0} \in C_{0}\left(\mathscr{M}_{I}\right)$ implies that $\left\|\hat{x}_{0}\right\|_{\mathscr{M}_{I}}<1$, and since $x_{0} \in I$ we have $x_{0} \in \mathscr{B}(A)$ by (1.2.v). And $\hat{x}_{0} \equiv \hat{x}^{m}$ on $U_{0}$.

Now, referring to (3.1) $\hat{I}_{e}$ contains a partition of unity subordinate to the covering $\left\{U_{0}, U_{1}, \ldots, U_{n}\right\}$ of $\mathscr{M}_{I}$ such that $\alpha_{1}, \ldots, \alpha_{n} \in I$. Also, there is no loss of generality in assuming here that $A$ has an identity, so that $I_{e} \subset A$. We may now apply (3.2) to this partition of unity and the elements $x_{0}, x_{1}^{n}, \ldots, x_{n}^{m}$ and conclude that $x^{m} \in \mathscr{B}(A)$, whence $x \in \mathscr{B}(A)$ by (1.2.iii).

(3.4) Corollary. Let $A$ be a semisimple, regular commutative Banach algebra, and let $x, y \in A$ with $r(x)=r(y)=1$. Suppose that $E_{x}=E_{y}$ and $\hat{x} \equiv \hat{y}$ on some neighborhood of $E_{x}\left(E_{x}\right.$ and $E_{y}$ are as in (4)). Then $x$ has bounded powers if and only if $y$ does.

(3.5) Corollary. Let A be a commutative, semisimple, regular Banach algebra. Then $x \in \mathscr{B}(A)$ is an extreme point of $\mathscr{B}(A)$ if and only if $\mathscr{M}_{A}$ is compact and $|\hat{x}| \equiv 1$ on $\mathscr{M}_{A}$.

Proof. Suppose first that $|\hat{x}| \equiv 1$. Since $\hat{x}$ is extreme in the unit ball of $C\left(\mathscr{M}_{A}\right)$, it follows that if $y \in A$ such that $x \pm y \in \mathscr{B}(A)$, then $\hat{y} \equiv 0$. Thus $y=0$, and $x$ is extreme.

Conversely, suppose $x$ is extreme in $\mathscr{B}(A)$ and $\left|\hat{x}\left(M_{0}\right)\right|<1$ for some $M_{0} \in \mathscr{M}_{A}$. By the regularity of $A$ we can find $y \in A$ such that $\hat{y}\left(M_{0}\right) \neq 0, \hat{y}=0$ on a neighborhood of $E_{x}$, and $|\hat{x}(M) \pm \hat{y}(M)|<1$ if $M \notin E_{x}$. Thus (3.4) applies to $x$ and $x \pm y$, and we conclude that $x \pm y \in \mathscr{B}(A)$, a contradiction.

4. The growth rates of iterates. For a semisimple commutative Banach algebra $A$ with discrete maximal ideal space we showed in (2.4) that any $x \in A$ is in $\mathscr{B}(A)$ unless the powers of $x$ grow exponentially to infinity. In this section we recall a class of algebras which serve to show that the rate at which the norms of powers of an element in a Banach algebra grow to infinity may be chosen essentially arbitrarily. In particular, it will follow that this sequence of powers may be made to grow to infinity "more slowly" than any preassigned sequence of numbers. This same class of algebras also provide us with the examples promised in the Remark of (1.2) above. 
(4.1) If $x$ is an element in a normed algebra and $\omega(n)=\left\|x^{n}\right\|, n=1,2, \ldots$, then the sequence $\omega$ satisfies

$$
\omega(m+n) \leqq \omega(m) \omega(n), \quad m, n=1,2, \ldots .
$$

Let $\Omega$ be the set of all positive sequences $\omega$ bounded away from zero and satisfying (5), and let $A_{1}$ be the algebra defined in (1.3). If $\omega \in \Omega$, let

$$
A_{\omega}=\left\{f \in A_{1}:\|f\|_{\omega}=\sum_{n=1}^{\infty}\left|a_{n}\right| \omega(n)<\infty\right\} \text {. }
$$

It is well known that $A_{\omega}$ is a semisimple commutative Banach algebra; similar algebras were first considered by A. Beurling in [1].

Proposition. There exists a commutative, semisimple Banach algebra $A_{\Omega}$ with the property that for every $\omega \in \Omega$ there is an $x \in A_{\Omega}$ such that $\left\|x^{n}\right\|=\omega(n), n=1,2, \ldots$

Proof. For $\omega \in \Omega,\left\|z^{n}\right\|_{\omega}=\omega(n), n=1,2, \ldots$ If we let $A_{\omega}=\bigoplus \sum_{\omega \in \Omega} A_{\omega}$, the normed full direct sum of the algebras $A_{\omega}$, then $A_{\Omega}$ is semisimple and has the desired properties.

(4.2) Corollary. Let $\left(\alpha_{n}\right)_{n=1}^{\infty}$ be any positive sequence tending to infinity. Then there exists $x \in A_{\Omega}$ such that $\left\|x^{n}\right\| \leqq C \alpha_{n}, n=1,2, \ldots$ and $\left\|x^{n}\right\| \rightarrow \infty$.

Proof. We must construct $\omega \in \Omega$ such that $\omega(n) \leqq C \alpha_{n}, n=1,2, \ldots$ and $\omega(n) \rightarrow \infty$. We proceed by first choosing positive numbers $C$ and $r$ so that $1<r^{2}<C \alpha_{n}, n=1$, $2, \ldots$. By induction one can choose a sequence $\left(n_{m}\right)_{m=1}^{\infty}$ of positive integers such that $C \alpha_{n} \geqq r^{m+1}$ if $n \geqq n_{m}$ and $n_{m}+n_{m^{\prime}} \leqq n_{m+m^{\prime}}, m, m^{\prime}=1,2, \ldots$ Let

$$
\omega(n)=r^{m+1} \text { if } n_{m} \leqq n<n_{m+1}, \quad m=1,2, \ldots
$$

An easy computation shows that $\omega$ satisfies (5).

(4.3) Proposition. Let $\omega \in \Omega$ such that $\omega(n) \rightarrow \infty$ but $\omega(n)^{1 / n} \rightarrow 1$. Then $\mathscr{B}\left(A_{\omega}\right)$ is open in $A_{\omega}$.

Proof. First note that, as in (1.3), $\mathscr{M}_{A_{\omega}} \cup\{0\}=\operatorname{sp}_{A_{\omega}}(z)=D$ because $r(z)=$ $\lim \left\|z^{n}\right\|_{\omega}^{1 / n}=\lim \omega(n)^{1 / n}=1$. Let $f \in A_{\omega}$ with $\|f\|_{D}=1$. Then $f=z g(z)$ with $g(z) \in\left(A_{1}\right)_{e}$, so

$$
\left\|f^{n}\right\|_{\omega}=\left\|z^{n} g^{n}\right\|_{\omega} \geqq\left\|z^{n}\right\|_{\omega}\left\|g^{n}\right\|_{1} \geqq \omega(n)\left\|g^{n}\right\|_{D}=\omega(n) \rightarrow \infty .
$$

(4.4) REMARK. It would seem to be of interest also to study the growth rates of iterates in algebras which are not semisimple. It can be shown, for example, that a result like (4.2) holds for certain algebras of operators. In fact, let $H$ be a separable Hilbert space, and for $\omega \in \Omega$ let $T$ be a right-hand weighted shift operator on $H$ with coefficients

$$
\omega(1), \frac{\omega(2)}{\omega(1)}, \frac{\omega(3)}{\omega(2)}, \ldots
$$

Then it is easy to see from (5) that $\left\|T^{n}\right\|=\omega(n), n=1,2, \ldots\left({ }^{3}\right)$.

$\left({ }^{3}\right)$ Pointed out to the author by L. J. Wallen. 
5. Measures on locally compact abelian groups.

(5.1) Notation. On a locally compact group $G, M(G)$ is the convolution algebra of finite, complex, regular Borel measures on $G$, the convolution of $\mu$, $\nu \in M(G)$ being written $\mu * \nu . M(G)$ is a Banach algebra under the total-variation norm; it contains as closed ideals the algebra $L^{1}(G)$ of measures absolutely continuous with respect to (left) Haar measure on $G$ (which we shall equate with the Borel-measurable functions integrable with respect to Haar measure) and the algebra $M_{c}(G)$ of continuous measures on $G$. Haar measure on $G$ will be denoted by $\lambda_{G}$, or $\lambda$ if $G$ is understood. $\mu_{d}$ is the discrete part of a measure on $G$, and the measure with mass one at the point $x \in G$ will be written $\delta_{x}$; in particular, $\delta_{e}$, where $e$ is the identity of $G$, is the identity of the algebra $M(G)$.

If $G$ contains a closed normal subgroup $H$ and if $\pi: G \rightarrow G / H$ is the natural homomorphism, we shall always assume that the Haar measures on $G, H$, and $G / H$ are normalized so that

$$
\int_{G} f(x) d \lambda_{G}(x)=\int_{G / H} f^{H}(\pi(x)) d \lambda_{G / H}(\pi(x)), \quad f \in L^{1}(G)
$$

where

$$
f^{H}(\pi(x))=\int_{H} f\left(x y^{-1}\right) d \lambda_{H}(y), \quad \lambda_{G / H^{-}} \text {a.e. }
$$

We shall also assume that Haar measures on compact groups are chosen to have total mass one and on discrete groups so that each point has mass one.

For a function $f$ defined on $G$ let $f_{x}$ be the function given by $f_{x}(y)=f\left(x^{-1} y\right)$, $y \in G$, and let

$$
\tilde{f}(x)=\overline{f\left(x^{-1}\right)} .
$$

$M(G)$ has a natural isometric involution given by

$$
\tilde{\mu}(E)=\overline{\mu\left(E^{-1}\right)}, \quad \mu \in M(G), \quad E \subset G .
$$

Also, for $\mu \in M(G)$ and $x \in G$ let $\mu_{x}$ be the measure $\mu_{x}(E)=\mu\left(x^{-1} E\right), E \subset G$.

If $G$ is a locally compact abelian group, we will use additive notation on $G$. The character group of $G$ will be called $\Gamma$, and the value of the character $\gamma$ at the point $x \in G$ is written $(x, \gamma)$. Of course, the Fourier-Stieltjes transform of a measure $\mu \in M(G)$ is denoted by $\hat{\mu}$, and the groups $Z, R$, and $T$ have their usual meaning. $G^{B}$ is our notation for the Bohr compactification of $G$, i.e., the character group of $\Gamma_{d}$, the group $\Gamma$ with the discrete topology. We shall assume that $\lambda_{G}$ and $\lambda_{\Gamma}$ are normalized so that the inversion formula [27, Theorem 1.5.1] holds. For further standard notation and results we refer to [15], [21], [26] and [36].

Finally, for any locally compact $G$ set $\mathscr{B}(M(G))=\mathscr{B}(G)$, and if $G$ is abelian and $\mu \in M(G)$ such that $\|\hat{\mu}\|_{\Gamma} \leqq 1$ we define $E_{\mu}$ in accord with (4) for the case $A=M(G)$ and $I=L^{1}(G)$ as

$$
E_{\mu}=\{\gamma \in \Gamma:|\hat{\mu}(\gamma)|=1\}
$$


If $x$ is in the center of a group $G$ and $\mu \in \mathscr{B}(G)$, then $\mu_{x}=\delta_{x} * \mu \in \mathscr{B}(G)$ by (1.2.ii). Furthermore, if $\gamma$ is a character of $G$ and $\mu \in \mathscr{B}(G)$, then $\gamma \cdot \mu \in \mathscr{B}(G)$ since multiplication by $\gamma$ is an isometric isomorphism of $M(G)$ with itself.

(5.2) We shall now mention two applications of our earlier results to measures on abelian groups; nonabelian groups will be dealt with in $\S 8$. The first is a direct application of (2.3). $|E|$ is the cardinality of a set $E$.

THEOREM. Let $G$ be a compact abelian group and $f \in L^{p}(G), 1 \leqq p<\infty$. Then $f \in \mathscr{B}\left(L^{p}(G)\right)$ if and only if $\|\hat{f}\|_{\Gamma} \leqq 1$. Furthermore, for such an $f$ we have

$$
\limsup _{n \rightarrow \infty}\left\|f^{n}\right\|_{p} \leqq\left|E_{f}\right| \text {. }
$$

Proof. Recall that $L^{p}(G) \subset L^{1}(G)$ and the Gelfand transform for the algebra $L^{p}(G)$ is just the Fourier transform (e.g., this follows from [21, 39E]). The first statement now follows from (2.3). Let $\|\hat{f}\|_{\Gamma} \leqq 1$, and let us use the notation of (2.2). If $E_{f}=\varnothing$, then $\left\|f^{n}\right\|_{p} \rightarrow 0$ since $r(f)<1$. If $E_{f} \neq \varnothing$, let $S_{i}=\left\{\gamma \in \Gamma: \hat{f}\left(\gamma_{i}\right)=\lambda_{i}\right\}$, $i=1, \ldots, n$. Then $e_{i}=\sum_{\gamma \in S_{i}} \gamma$, and since $\|\gamma\|_{p}=1, \gamma \in \Gamma$, we get $\left\|e_{i}\right\|_{p} \leqq\left|S_{i}\right|$. The last inequality in the proof of (2.2) then gives

$$
\limsup _{k \rightarrow \infty}\left\|f^{k}\right\|_{p} \leqq \sum_{i=1}^{n}\left\|e_{i}\right\|=\sum_{i=1}^{n}\left|S_{i}\right|=\left|E_{f}\right| \text {. }
$$

(5.3) Theorems (3.2) and (3.3) apply to the algebras $A=M(G)$ and $I=L^{1}(G)$. Moreover, in this case $M(G)^{\wedge}$ contains partitions of unity subordinate to coverings $\left\{U_{1}, \ldots, U_{n}\right\}$ of $\Gamma$ of a more general nature than those discussed in (3.4), i.e., without the assumption that some $U_{i}^{\prime}$ is compact in $\Gamma$. For such coverings we then have, by the proof of (3.2) and the uniqueness of Fourier-Stieltjes transforms, that if $\mu_{1}, \ldots, \mu_{n} \in \mathscr{B}(G)$ such that $\hat{\mu}_{i} \equiv \hat{\mu}_{j}$ on $U_{i} \cap U_{j}, i, j=1, \ldots, n$ there is a unique $\mu \in \mathscr{B}(G)$ with $\hat{\mu} \equiv \hat{\mu}_{i}$ on $U_{i}, i=1, \ldots, n$. For example, if $\beta: \Gamma \rightarrow \Gamma^{B}$ is the natural injection, $\left\{\tilde{U}_{1}, \ldots, \tilde{U}_{n}\right\}$ is an open covering of $\Gamma^{B}$, and $U_{i}=\beta^{-1}\left(\tilde{U}_{i}\right), i=1, \ldots, n$, then $\hat{M}_{d}$ contains a partition of unity subordinate to $\left\{U_{1}, \ldots, U_{n}\right\}$. Another class of such coverings is given in the following theorem.

THEOREM. For $i=1,2, \ldots, n$, let $\Lambda_{i}$ be a closed subgroup of $\Gamma$ and $\pi_{i}: \Gamma \rightarrow \Gamma / \Lambda_{i}$ the natural map. Suppose that $V_{i}$ and $Y_{i}$ are open sets in $\Gamma / \Lambda_{i}$ such that $V_{i} \cup Y_{i}$ $=\Gamma / \Lambda_{i}$ and either $V_{i}^{\prime}$ or $Y_{i}^{\prime}$ is compact in $\Gamma / \Lambda_{i}$. Let $U_{0}=\bigcap_{i=1}^{n} \pi_{i}^{-1}\left(Y_{i}\right)$ and $U_{i}$ $=\pi_{i}^{-1}\left(V_{i}\right), i=1, \ldots, n$. Then $\left\{U_{0}, U_{1}, \ldots, U_{n}\right\}$ is an open covering of $\Gamma$ which has a subordinate partition of unity contained in $M(G)^{\wedge}$.

Proof. That $\left\{U_{0}, U_{1}, \ldots, U_{n}\right\}$ is a covering is clear. Let $H_{i}$ be the annihilator of $\Lambda_{i}$ in $G$, so that $\Gamma / \Lambda_{i}$ is the character group of $H_{i}, i=1, \ldots, n$. If $Y_{i}^{\prime}$ is compact, then since $Y_{i}^{\prime} \subset V_{i}$ we can choose $p_{i} \in L^{1}\left(H_{i}\right)$ such that $\hat{p}_{i} \equiv 1$ on $Y_{i}^{\prime}, \hat{p}_{i} \equiv 0$ on $V_{i}^{\prime}$, and $0 \leqq \hat{p}_{i} \leqq 1$. (Here $\hat{p}_{i}$ is the Fourier transform of $p_{i}$ as a function on $\Gamma / \Lambda_{i}$.) If $V_{i}^{\prime}$ is compact we choose $p_{i}=\delta_{0}-p_{i}^{\prime}$ where $p_{i}^{\prime} \in L^{1}\left(H_{i}\right)$ satisfying $\hat{p}_{i}^{\prime} \equiv 1$ on $V_{i}^{\prime}$, $\hat{p}_{i}^{\prime} \equiv 0$ on $Y_{i}^{\prime}$, and $0 \leqq \hat{p}_{i}^{\prime} \leqq 1$; the $\hat{p}_{i}$ then have the same properties as those chosen in the previous case. 
Now consider $L^{1}\left(H_{i}\right)$ as a subalgebra of $M(G)$ for each $i$, so that $p_{i}$ corresponds to a measure $\mu_{i}$ whose Fourier-Stieltjes transform is $\hat{\mu}_{i}=\hat{p}_{i} \circ \pi_{i}, i=1, \ldots, n$. Then $\gamma \notin U_{i}$ implies $\hat{\mu}_{i}(\gamma)=\hat{p}_{i}\left(\pi_{i}(\gamma)\right)=0, \quad i=1, \ldots, n$. Let $\mu_{0}=\left(\delta_{0}-\mu_{1}\right) * \cdots *$ $\left(\delta_{0}-\mu_{n}\right)$. If $\gamma \notin U_{0}$, then $\gamma \notin \pi_{k}^{-1}\left(Y_{k}\right)$ for some $k \leqq n$ and hence $\hat{p}_{k}\left(\pi_{k}(\gamma)\right)=1$.

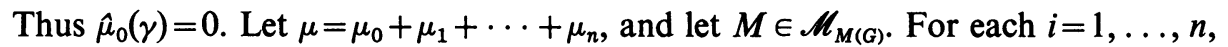
either $L^{1}\left(H_{i}\right) \subset M$ or we can find $\bar{\gamma}_{i} \in \Gamma / \Lambda_{i}$ such that $\hat{\sigma}(M)=\hat{f}\left(\bar{\gamma}_{i}\right)$ whenever $d \sigma$ $=f d \lambda_{H_{i}}$ for some $f \in L^{1}\left(H_{i}\right)$. In particular, for $i=1, \ldots, n$ we have either $\hat{\mu}_{i}(M)=0$ or $\hat{\mu}_{i}(M)=\hat{p}_{i}\left(\bar{\gamma}_{i}\right)$, so that $0 \leqq \hat{\mu}_{i}(M) \leqq 1$. Thus $0 \leqq\left(\delta_{0}-\mu_{i}\right)^{\wedge}(M)=1-\hat{\mu}_{i}(M) \leqq 1$, $i=1, \ldots, n$, and hence $0 \leqq \hat{\mu}_{0}(M) \leqq 1$ also. If $\hat{\mu}(M)=\hat{\mu}_{0}(M)+\hat{\mu}_{1}(M)+\cdots+\hat{\mu}_{n}(M)$ $=0$, we must have $\hat{\mu}_{0}(M)=\hat{\mu}_{1}(M)=\cdots=\hat{\mu}_{n}(M)=0$. But $\hat{\mu}_{0}(M)=0$ implies that $\hat{\mu}_{i}(M)=1$ for some $i \geqq 1$. Thus $\hat{\mu}(M) \neq 0$ for all $M \in \mathscr{M}_{M(G)}$ so $\mu$ is invertible in $M(G)$. The required partition of unity is thus given by the measures $\alpha_{i}=\mu^{-1} * \mu_{i}$, $i=0,1, \ldots, n$.

6. The study of $\mathscr{B}(G)$. We begin this section with our main theorem on locally compact abelian groups, a necessary condition (6.2) for a set $E \subset \Gamma$ to be of the form $E_{\mu}$ and for a function on $E$ to be the restriction of $\hat{\mu}$, for some $\mu \in \mathscr{B}(G)$. In particular, we may conclude from this description that the measures $\mu \in \mathscr{B}(G)$ with $E_{\mu} \neq \varnothing$ are a relatively small subset of the set of measures $\mu \in M(G)$ satisfying $\|\hat{\mu}\|_{\Gamma}=1$. A number of applications of this theorem follow, including a rather unsatisfying characterization of $\mathscr{B}(G)$, and we conclude with some results on a certain lifting problem.

Recall that the coset ring $\mathscr{R}(G)$ of an abelian group $G$ is the smallest Boolean algebra of subsets of $G$ containing the cosets of all subgroups of $G$. If $K$ is a coset in $G, \phi: K \rightarrow G^{*}$, and $x_{0} \in K$, then $\phi$ is called affine if there exists a homomorphism $\phi:\left(K-x_{0}\right) \rightarrow G^{*}$ such that $\phi(x)=\phi\left(x-x_{0}\right)+\phi\left(x_{0}\right), x \in K$. If $S \in \mathscr{R}(G)$ and $\phi: S \rightarrow G^{*}$, then $\phi$ is called piecewise affine on $S$ if we can find $S_{1}, \ldots, S_{n} \in \mathscr{R}(G)$, cosets $K_{1}, \ldots, K_{n}$ in $G$, and affine maps $\phi_{i}: K_{i} \rightarrow G^{*}, i=1, \ldots, n$, such that $S_{i} \subset K_{i}, \phi=\phi_{i}$ on $S_{i}, i=1, \ldots, n$, and $S=S_{1} \cup \ldots \cup S_{n}$.

(6.1) Lemma. Let $G$ be a compact abelian group and $\mu \in \mathscr{B}(G)$. Then $E_{\mu} \in \mathscr{R}(\Gamma)$ and $\hat{\mu} \mid E_{\mu}$ is a piecewise affine map of $E_{\mu}$ into $T$.

Proof. Let $\sigma$ be a weak-* cluster point of the sequence $\left(\mu^{n}\right)_{n=1}^{\infty}(\sigma$ exists since $\mu \in \mathscr{B}(G))$. Then

$$
\begin{aligned}
|\hat{\sigma}(\gamma)|=\lim _{n \rightarrow \infty}\left|\hat{\mu}(\gamma)^{n}\right| & =1 & & \gamma \in E_{\mu} \\
& =0 & & \gamma \notin E_{\mu}, \quad \gamma \in \Gamma .
\end{aligned}
$$

Setting $\eta=\sigma * \tilde{\sigma}$, we have $\hat{\eta}=|\hat{\sigma}|^{2}=\chi_{E_{\mu}}$; by a well-known result of Cohen [6], [26, Theorem 3.1.3, p. 60] (see also [18]), $E_{\mu} \in \mathscr{R}(\Gamma)$.

Let $\nu=\mu * \eta$, so that $|\hat{\nu}| \equiv 1$ on $E_{\mu}$. Then $\nu, \tilde{\nu} \in \mathscr{B}(G)$ and $\nu * \tilde{\nu}=\eta\left(\right.$ for $(\nu * \tilde{\nu})^{\wedge}$ $\left.=\hat{\nu} \hat{\nu}^{-}=|\hat{\mu}|^{2} \hat{\eta}=\hat{\eta}\right)$. Define $T: L^{1}(Z) \rightarrow M(G)$ by

$$
T f=\sum_{n=1}^{\infty} f(-n) \nu^{n}+f(0) \eta+\sum_{n=1}^{\infty} f(n) \tilde{\nu}^{n}, \quad f \in L^{1}(Z) .
$$


A computation shows that $T$ is an algebra homomorphism (cf. (1.3)). Moreover,

$$
\begin{array}{rlrl}
(T f)^{\wedge}(\gamma) & =\sum_{n=1}^{\infty} f(-n) \hat{v}(\gamma)^{n}+f(0) \hat{\eta}(\gamma)+\sum_{n=1}^{\infty} f(n) \overline{\hat{v}(\gamma)^{n}} \\
& =\sum_{n \in Z} f(n)(\hat{\mu}(\gamma))^{-n} & & \gamma \in E_{\mu} \\
& =0 & & \gamma \notin E_{\mu} \\
& =\hat{f}(\hat{\mu}(\gamma)) & & \gamma \in E_{\mu} \\
& =0 & & \gamma \notin E_{\mu}, \quad f \in L^{1}(Z), \quad \gamma \in \Gamma .
\end{array}
$$

It now follows from Cohen's theorem on homomorphisms of group algebras [7], [26, Theorem 4.1 .3$, p. 78] that $\hat{\mu} \mid E_{\mu}$ is piecewise affine.

(6.2) THEOREM. Let $G$ be a locally compact abelian group and $\mu \in \mathscr{B}(G)$. There exist subsets $E_{1}, \ldots, E_{n}$ of $\Gamma, x_{1}, \ldots, x_{n} \in G$, and $c_{1}, \ldots, c_{n} \in T$ such that

(i) $E_{\mu}=E_{1} \cup \cdots \cup E_{n}$.

(ii) For each $1 \leqq k \leqq n, E_{k}$ has the form

$$
\bigcup_{j=1}^{N}\left(\Lambda_{0}^{j} \mid \bigcup_{i=1}^{n(j)} \Lambda_{i}^{j}\right)
$$

where the $\Lambda_{i}^{j}$ are closed (possibly void) cosets in $\Gamma$ such that for each $j=1, \ldots, N$ $\Lambda_{i}^{j}$ is relatively open in $\Lambda_{0}^{j}, j=1, \ldots, n(j)$.

(iii) $\hat{\mu}(\gamma)=c_{k}\left(x_{k}, \gamma\right), \gamma \in E_{k}, k=1, \ldots, n$.

Proof. We may consider $\mu$ as a measure on $G^{B}$, in fact an element of $\mathscr{B}\left(G^{B}\right)$, in the usual way. Lemma (6.1) then implies that as a subset of $\Gamma_{d} E_{\mu}=S_{1} \cup \cdots \cup S_{N}$ as in (6) and that there are affine maps $\phi_{j}: \Lambda_{0}^{j} \rightarrow T$ such that $\hat{\mu} \equiv \phi_{j}$ on $S_{j}, j=1, \ldots$, $n$. Now, $E_{\mu}$ is closed since $\hat{\mu}$ is continuous, so $E_{\mu}=\bar{S}_{1} \cup \cdots \cup \bar{S}_{n}$. Let $E_{k}=\bar{S}_{k}$, $k=1, \ldots, n$. Then it was shown in [10], [28] that each of the $E_{k}$ has the form (6) as a subset of $\Gamma$. Moreover, in defining the $S_{j}$ we may assume that for each $j$ and each $i \geqq 1 \quad \Lambda_{i}^{j}$ has infinite index in $\Lambda_{0}^{j}$ (in the obvious sense). It then follows (see [7, p. 223]) that since $\hat{\mu}$ is continuous each $\phi_{j}$ is uniformly continuous on $\Lambda_{0}^{j}$ and hence can be extended to a continuous affine map $\bar{\phi}_{j}: \bar{\Lambda}_{0}^{j} \rightarrow T$. It is clear that such a map is merely a character of $\Gamma$ multiplied by a unimodular constant; for the homomorphism associated with the map $\tilde{\phi}_{j}$ is just a character on a closed subgroup of $\Gamma$, which can be extended to a character of $\Gamma$, giving $\phi_{j}$ the form (iii) by Pontryagin duality. Since $\hat{\mu} \equiv \phi_{k}$ on $E_{k}$, the theorem is proved.

(6.3) Corollary. (i) Let $\mu \in \mathscr{B}(G)$ and $c_{k}, x_{k}$ as in (6.2), and let the $E_{k}$ be chosen so that $E_{k}=\left\{\gamma: \hat{\mu}(\gamma)=c_{k}\left(x_{k}, \gamma\right)\right\}, k=1, \ldots, n$. Then for each $k E_{k}=E_{v}$ for some $\nu \in \mathscr{B}(G)$ such that $\hat{v}(\gamma)=c_{k}\left(x_{k}, \gamma\right), \gamma \in E_{k}$.

(ii) Let $\mu \in \mathscr{B}(G), c \in T, x \in G$. Then there exists $\nu \in \mathscr{B}(G)$ such that $E_{v}=E_{\mu}$ and $\hat{v}(\gamma)=c(x, \gamma), \gamma \in E_{\mu}$. 
Proof. (i) Fix $k$ and let $\nu=\frac{1}{2}\left(\mu+c_{k} \delta_{x_{k}}\right)$. Then $\nu \in \mathscr{B}(G)$ by (1.2.i) and $\hat{\nu}(\gamma)=$ $c_{k}\left(x_{k}, \gamma\right), \gamma \in E_{k}$. If $|\hat{\nu}(\gamma)|=\frac{1}{2}\left|\hat{\mu}(\gamma)+c_{k}\left(x_{k}, \gamma\right)\right|=1$, then $\gamma \in E_{k}$ since $|\hat{\mu}(\gamma)| \leqq 1$.

(ii) Let $\nu=c \delta_{x} * \mu * \tilde{\mu}$, and it is clear that $\nu$ has the required properties for (ii).

(6.4) CoRollary. Let $f \in L^{1}(G) \cap \mathscr{B}(G)$. Then $E_{f}$ and $\hat{f} \mid E_{f}$ are as described in (6.2) with each $E_{k}$ being a compact coset in $\Gamma$.

Proof. For $f \in L^{1}(G) E_{f}$ is compact. It was shown in [28, Corollary (1.8)] that a compact subset of $\mathscr{R}(G)$ is a finite union of compact cosets.

(6.5) Corollary. Let $G$ be connected and $f \in L^{1}(G) \cap \mathscr{B}(G)$. Then $E_{f}$ is finite.

Proof. If $G$ is connected $\Gamma$ is topologically isomorphic with $R^{n} \times F$, where $n$ is a nonnegative integer and $F$ is a discrete torsion-free abelian group [15, Corollary (24.35), p. 390]. Since $E_{f}$ is compact there is a finite set $\left\{\gamma_{1}, \ldots, \gamma_{k}\right\} \subset F$ such that $E_{f} \subset R^{n} \times\left\{\gamma_{1}, \ldots, \gamma_{k}\right\}$. Since $F$ is torsion free, the group generated by $\gamma_{1}, \ldots, \gamma_{k}$ is isomorphic with $Z^{m}$ for some nonnegative integer $m$. Thus $E_{f}$ may be considered as a member of $\mathscr{R}\left(R^{n} \times Z^{m}\right)$. The result now follows from (6.4) and the fact that the only compact subgroup of $R^{n} \times Z^{m}$ is $\{0\}$.

(6.6) Corollary [16, Theorem 2.4]. Let $\mu \in M(G)$ with $\|\mu\|=1$. Then $E_{\mu}$ is a (perhaps void) closed coset in $\Gamma$.

Proof. Let $\eta$ be the idempotent in $M\left(G^{B}\right)$ such that $\hat{\eta}=\chi_{E_{\mu}}$. Then as shown in the proof of (6.1), if $\eta \neq 0$ then $\|\eta\|=1$. Whence $E_{\eta}$ is a coset in $\Gamma$.

(6.7) THEOREM. If $|\hat{\mu}| \equiv 1$ on $\Gamma$, then $\mu \in \mathscr{B}(G)$ if and only if $\Gamma$ and $\hat{\mu}$ are as in (6.2). Moreover, in this case the $\Lambda_{0}^{j}$ of (6), whence all the $E_{k}$, may be chosen open in $\Gamma$.

Proof. Suppose that $\mu \in \mathscr{B}(G)$, and write $E_{\mu}(=\Gamma)$ and $\hat{\mu}$ as in (6.2). Then we have $\Gamma$ as a union of the form (6), and each set in this union has nonvoid interior if and only if it is open and closed. The Baire category theorem now tells us that $\Gamma$ is in fact covered by the open and closed sets in the union, and we may disregard any others. Finally, note that any open and closed set in $\mathscr{R}(G)$ may be written in the form (6) with each $\Lambda_{i}^{j}, i \geqq 0$, being open and closed [7, pp. 223-224], [28, (1.2)].

Conversely, let $\eta_{k}$ be idempotent in $M(G)$ with $\hat{\eta}_{k}=\chi_{E_{k}}, k=1, \ldots, n$. Then

$$
\begin{aligned}
\mu & =\sum_{k=1}^{n} c_{k} \delta_{x_{k}} * \eta_{k}, \\
\mu^{m}=\sum_{i=1}^{n} c_{i}^{m} \delta_{x_{i}}^{m} * \eta_{i} & =\sum_{i=1}^{n} c_{i}^{m} \delta_{m x_{i}} * \eta_{i}, \quad m=1,2, \ldots,
\end{aligned}
$$

giving $\left\|\mu^{m}\right\| \leqq \sum_{k=1}^{n}\left\|\eta_{k}\right\|, m=1,2, \ldots$

REMARK. Since $\mu^{*}=\mu^{-1}$ if $|\hat{\mu}| \equiv 1$, this result is just a restatement of [26, Theorem 4.7.3, p. 93]. 
(6.8) Corollary. Let $f \in L^{1}(G)$ such that $\|\hat{f}\|_{\Gamma} \leqq 1$ and $E_{f}$ is open in $\Gamma$. Then $f \in \mathscr{B}(G)$ if and only if there exist cosets $\Delta_{1}, \ldots, \Delta_{n}$ of some compact open subgroup $\Lambda$ of $\Gamma, c_{1}, \ldots, c_{n} \in T$, and $x_{1}, \ldots, x_{n} \in G$ such that

(i) $E_{f}=\Delta_{1} \cup \ldots \cup \Delta_{n}$,

(ii) $\hat{f}(\gamma)=c_{i}\left(x_{i}, \gamma\right), \gamma \in \Delta_{i} ; i=1, \ldots, n$.

Proof. Let $f \in \mathscr{B}(G)$. If we apply (6.4) and argue as in (6.7) we obtain $E_{f}$ as a finite union of open compact cosets. (i) follows, $\Lambda$ being the intersection of the corresponding open subgroups.

Conversely, suppose (i) and (ii) hold, and let $H$ be the annihilator of $\Lambda$ in $G$, a compact open subgroup of $G$. Assume that $\lambda_{G}$ is chosen so that $\lambda_{G}(H)=\left\|\chi_{H}\right\|_{1}=1$, so $\chi_{H}=\chi_{\Lambda}$. It follows that we may write $f=g+h$, where $g, h \in L^{1}(G), g * h=0, g$ has the form (7), and $\|\hat{h}\|_{\Gamma}<1$. Thus $f \in \mathscr{B}(G)$ (cf. (2.3)).

(6.9) ExAMPLE. Let $G$ denote $Z\left(p^{\infty}\right)$ or the additive structure of the $p$-adic number field $\Omega_{p}$, for some prime $p$. Let $f \in L^{1}(G)$ such that $\|\hat{f}\|_{\Gamma} \leqq 1, \hat{f}$ is piecewise affine on $E_{f} \in \mathscr{R}(\Gamma)$, and $E_{f}$ is perfect. Then $f \in \mathscr{B}(G)$.

Proof. We have shown in (6.2) that $\hat{f}$ piecewise affine on $E_{f} \in \mathscr{R}(\Gamma)$ implies that $\hat{f} \mid E_{f}$ has the form described in that theorem. Recall that the character group of $Z\left(p^{\infty}\right)$ is the open subgroup $\Delta_{p}$ of $\Omega_{p}$ of $p$-adic integers and that $\Omega_{p}$ is self-dual [15, (25.1), (25.2), pp. 400-403]. The rest follows from (6.8) and the fact that perfect subsets of $\mathscr{R}\left(\Omega_{p}\right)$ are open [28, (1.10)].

(6.10) COROLlary. $\mu$ is an extreme point of $\mathscr{B}(G)$ if and only if $\hat{\mu}$ has the form described in (6.7). Thus if $G$ is nondiscrete then $\mathscr{B}\left(L^{1}(G)\right)$ has no extreme points, while if $\Gamma$ is connected the extreme points of $\mathscr{B}(G)$ are precisely the measures $c \delta_{x}$, $|c|=1, x \in G$.

Proof. This is easily recognized as a variant of (3.5).

REMARK. It is interesting to compare this result with the well-known facts that the extreme points of the unit ball of $M(G)$ are just the unimodular multiples of point-masses, and hence if $G$ is nondiscrete the unit ball of $L^{1}(G)$ has no extreme points.

(6.11) Proposition. Let $\mu \in M(G)$ and let $\Lambda$ be a closed subgroup of $\Gamma$ such that $\Gamma / \Lambda$ is compact. If $|\hat{\mu}(\gamma)| \equiv 1$ on $\gamma_{0}+\Lambda$, then $\mu_{d} \neq 0$.

Proof. Let $d \nu(x)=\left(-x, \gamma_{0}\right) d \mu(x)$ and $\sigma=\nu * \tilde{\nu}$. Then $\hat{\sigma}(\gamma)=|\hat{v}(\gamma)|^{2}=\left|\hat{\mu}\left(\gamma+\gamma_{0}\right)\right|^{2}$ $=1, \gamma \in \Lambda$. Let $H$ be the annihilator of $\Lambda$ in $G$. Then the map of $M(G)$ onto $M(G / H)$ (which restricts Fourier-Stieltjes transforms) induced by the natural map of $G$ onto $G / H$ maps $\sigma$ to $\delta_{0}$. Thus $|\sigma|(H) \geqq \delta_{0}(\{0\})=1$. Since $\Gamma / \Lambda$ is compact $H$ is discrete, so $\sigma_{d} \neq 0$. Now, $\sigma_{d}=\nu_{d} * \tilde{\nu}_{d}$, so $\nu_{d} \neq 0$; and $d \nu_{d}(x)=\left(-x, \gamma_{0}\right) d \mu_{d}(x)$, whence $\mu_{d} \neq 0$.

(6.12) Corollary. Let $G=R$ or $T$ and $\mu \in M_{c}(G) \cap \mathscr{B}(G)$. Then $E_{\mu}$ is finite.

(6.13) Proposition. Let $\mu \in \mathscr{B}(G)$, and suppose that for some compact $K \subset G$ and infinitely many $n$, support $\left(\mu^{n}\right) \subset K$. Then $E_{\mu}$ is open in $\Gamma$. 
Proof. For notational purposes let us assume the hypothesis holds for all $n$. Let $\nu$ be a weak-* limit point of $\left((\mu * \tilde{\mu})^{n}\right)_{n=1}^{\infty}$, all of which have support contained in the compact set $K-K=K^{*}$. Since $\gamma \mid K^{*} \in C\left(K^{*}\right), \gamma \in \Gamma$, we have

$$
\hat{v}(\gamma)=\lim _{n \rightarrow \infty}|\hat{\mu}(\gamma)|^{2 n}=\chi_{E_{\mu}}(\gamma)
$$

which must be continuous. Thus $E_{\mu}$ is open.

(6.14) Definition. Let $\mathscr{E}(\Gamma)=\left\{E_{\mu}: \mu \in \mathscr{B}(G)\right\}$. We have shown in (6.2) that every set in $\mathscr{E}(\Gamma)$ is in $\mathscr{R}(\Gamma)$ and, in fact, has the form (6). We now obtain, in (6.20), a partial converse to this statement. It is clear that $\mathscr{E}(\Gamma)$ is closed under translations and finite intersections, that every open set in $\mathscr{R}(\Gamma)$ is in $\mathscr{E}(\Gamma)$, and that every $E_{\mu}$ is a $G_{o}$-set.

(6.15) LEMMA. Let $G$ be a locally compact group and $H$ a closed normal subgroup of $G$. Then $G / H$ is metrizable if and only if $H$ is $a G_{\delta}$ in $G$.

Proof. Suppose $V_{1} \supset V_{2} \supset \cdots$ are open sets in $G$ such that $H=\bigcap_{1}^{\infty} V_{i}$. Choose an open set $W_{1} \subset V_{1}$ containing $e_{G}$ such that $\bar{W}_{1}$ is compact, and choose inductively $W_{2}, W_{3}, \ldots$ open sets with $e_{G} \in W_{i} \subset \bar{W}_{i} \subset W_{i-1} \cap V_{i}, i=2,3, \ldots$ Let $U_{i}=\pi\left(W_{i}\right)$, $i=1,2, \ldots$ Then $e_{G / H} \in \bigcap_{1}^{\infty} U_{i}$; let $x \in \bigcap_{1}^{\infty} U_{i}$. For each $i$ we may choose $w_{i} \in W_{i}$ so that $\pi\left(w_{i}\right)=x$. Since $w_{i} \in \bar{W}_{i}, i=1,2, \ldots$, the sequence of $w_{i}$ has a convergent subnet, say $w_{\alpha} \rightarrow y$. And since $\bigcap_{1}^{\infty} W_{i} \subset \bigcap_{1}^{\infty} V_{i}=H$ and every subnet of $\left(w_{i}\right)_{i=1}^{\infty}$ must ultimately be in every $\bar{W}_{i}$, we see that $y \in H$. Thus $x=\lim _{\alpha} \pi\left(w_{\alpha}\right)=\pi(y)=e_{G \mid H}$, and we have $\left\{e_{G / H}\right\}=\bigcap_{1}^{\infty} U_{i}$, so $G / H$ is metrizable [15, Theorem (8.5), pp. 70-71].

The converse is obvious.

(6.16) LemMA. Let $G$ be a locally compact abelian group and $\Lambda$ a closed $G_{\delta}$-subgroup of $\Gamma$, and let $U$ be a neighborhood of 0 in $\Gamma$. Then there exists $\mu \in M(G)$ such that $\|\mu\|=1, E_{\mu}=\Lambda$, and $\hat{\mu} \equiv 0$ off $\Lambda+U$.

Proof. Let $H$ be the annihilator of $\Lambda$ in $G$. By (6.15) the dual $\Gamma / \Lambda$ of $H$ is metrizable. We may choose $f \in L^{1}(H)$ of norm one with $E_{f}=\{0\}$ and $\hat{f} \equiv 0$ off $\pi(U)=V$. (In fact, let $V \supset V_{1} \supset V_{2} \supset \cdots$ be open in $\Gamma / \Lambda$ with $\bigcap_{1}^{\infty} V_{i}=\{0\}$, and for each $i$ choose $f_{i} \in L^{1}(H)$ such that $\hat{f}_{i}(0)=1, \hat{f}_{i} \equiv 0$ outside $V_{i}$, and $\left\|f_{i}\right\|_{1}=1$. Then $f=\sum_{1}^{\infty} 2^{-i} f_{i}$ is the required function.) Then considering $d \mu=f d \lambda_{H}$ as a measure in $M(G)$ we have the desired measure $\left({ }^{4}\right)$.

(6.17) LEMMA. Let $\Lambda$ be a closed $G_{\delta}$-subgroup of $\Gamma$ and $\Delta$ a relatively open subgroup of $\Lambda$, and let $E=\Lambda \mid \Delta$. If $V$ is a neighborhood of 0 in $\Gamma$ there exists $\mu \in \mathscr{B}(G)$ such that $E_{\mu}=E$ and $\hat{\mu}(\gamma)=0, \gamma \notin E+V$.

Proof. Let $H$ and $K$ be the annihilators in $G$ of $\Lambda$ and $\Delta$, respectively. Let $\pi_{1}: \Gamma \rightarrow \Gamma / \Delta, \pi_{2}: \Gamma \rightarrow \Gamma / \Lambda$, and $\pi: \Gamma / \Delta \rightarrow \Gamma / \Lambda$ be defined in the natural way so

(4) Lemma (6.15) is not new; for lack of a reference we have included the proof. Lemma (6.16) is a restatement of [16, Theorem 1.1], though the proof is somewhat different. We shall require the form of the measure $\mu$ (as constructed in our proof) in (6.17). 
that $\pi \circ \pi_{1}=\pi_{2}$. Note that $\pi$ is a local homeomorphism since $\Lambda / \Delta$ is discrete in $\Gamma / \Delta$. Let $U=\pi_{1}(V)$ be chosen so that it is a neighborhood of 0 in $\Gamma / \Delta$ on which $\pi$ is a homeomorphism. As in (6.16) we may find $f \in L^{1}(H)$ such that $\|f\|_{1}=1$, $E_{f}=\{0\}$, and $\hat{f} \equiv 0$ off $\pi(U)$. Similarly, we may choose $g \in L^{1}(K)$ with $\|g\|_{1}=1$, $E_{g}=\{0\}$, and $\hat{g} \equiv 0$ off $U$. Since $\pi$ is a local isomorphism we may further assume that $\hat{g}(\bar{\gamma})=\hat{f} \circ \pi(\bar{\gamma}), \bar{\gamma} \in U$. That is to say, $\hat{f} \circ \pi$ is the periodic extension of $\hat{g}$ with respect to the group $\Lambda / \Delta$. (For example, assume $\lambda_{\Gamma / \Delta}=\lambda_{\Gamma / \Lambda} \circ \pi$ on $U$ and proceed as in (6.16), noting that for $h \in L^{1}(\Gamma / \Lambda)$ with support a sufficiently small neighborhood of 0 we have $(h * \tilde{h}) \circ \pi=k * \tilde{k}$, where $k \equiv h \circ \pi$ on $U$ and $k \equiv 0$ off $U$. Thus if $f_{i}$ and $g_{i}$ are analogous to the $f_{i}$ in (6.16) let them be of the form $\hat{f}_{i}=h * \tilde{h}$ and $\hat{g}_{i}=k * \tilde{k}$ for an appropriate choice of $h$.) Let $d \mu=f d \lambda_{H}-g d \lambda_{K}$. Then

$$
\begin{array}{rlrl}
\hat{\mu}(\gamma) & =\hat{f} \circ \pi_{2}(\gamma)-\hat{g} \circ \pi_{1}(\gamma)=(\hat{f} \circ \pi-\hat{g}) \circ \pi_{1}(\gamma) \\
& =\hat{f} \circ \pi_{2}(\gamma) & \pi_{1}(\gamma) \notin U \\
& =0 & & \pi_{1}(\gamma) \in U .
\end{array}
$$

Thus $E_{\mu}=\Lambda \cap \pi_{1}^{-1}(U)^{\prime}=\Lambda \mid \Delta$. Since $\hat{\mu}^{n}=\hat{f}^{n} \circ \pi_{2}-\hat{g}^{n} \circ \pi_{1}, n=1,2, \ldots$, we see that $\mu \in \mathscr{B}(G)$. If $\hat{\mu}(\gamma) \neq 0$, then $\pi_{1}(\gamma) \notin U=\pi_{1}(V)$ and $\pi_{2}(\gamma)=\pi \circ \pi_{1}(\gamma) \in \pi(U)=\pi \circ \pi_{1}(V)$ $=\pi_{2}(V)$. Thus

$$
\gamma \in \pi_{2}^{-1}\left(\pi_{2}(V)\right)\left|\pi_{1}^{-1}\left(\pi_{1}(V)\right)=(\Lambda+V)\right|(\Delta+V) \subset E+V .
$$

(6.18) Definition. Let $G$ be an abelian topological group, and let $E$ and $F$ be closed sets in $G$. $E$ and $F$ will be called uniformly separated if there is a neighborhood $U$ of 0 such that $(E+U) \cap F=\varnothing$. Notice that if $V$ is a symmetric neighborhood of 0 such that $V+V \subset U$, then $(E+V) \cap(F+V)=\varnothing$.

(6.19) Proposition. Let $E$ and $F$ be closed sets in $G$. $E$ and $F$ are uniformly separated if either

(i) $E$ is compact and disjoint from $F$, or

(ii) $E \subset H_{1}$ and $F \subset H_{2}, H_{1}$ and $H_{2}$ being distinct cosets of some closed subgroup $H$ of $G$.

(6.20) THEOREM. Let $E=E_{1} \cup \cdots \cup E_{N}$ be a closed $G_{\delta}$-set in $\mathscr{R}(\Gamma)$ written in the form (6), and suppose that the $E_{k}$ are pairwise uniformly separated. Then $E \in \mathscr{E}(\Gamma)$.

Proof. Let us first assume $N=1$, so that $E=\Lambda \mid \bigcup_{1}^{n} \Delta_{i}$ as in (6). Applying (6.17) and translating, we see that each of the sets $S_{i}=\Lambda \mid \Delta_{i}$ is in $\mathscr{E}(\Gamma)$, and hence $E=S_{1}$ $\cap \cdots \cap S_{n} \in \mathscr{E}(\Gamma)$. Moreover, we can find a neighborhood $V$ of 0 in $\Gamma$ such that $\left(S_{i}+V\right)=(\Lambda+V) \backslash\left(\Delta_{i}+V\right), i=1, \ldots, n$. Then

$$
E+V \subset \bigcap_{i=1}^{n}\left(S_{i}+V\right)=(\Lambda+V) \backslash \bigcup_{i=1}^{n}\left(\Delta_{i}+V\right) \subset E+V,
$$

so $E+V=\bigcap_{1}^{n}\left(S_{i}+V\right)$. Now applying (6.17) we see that the conclusions of (6.17) hold for $E$ and this $V$. 
We may now treat the general case. Let $V$ be a neighborhood of 0 such that

(i) $\left(E_{i}+V\right) \cap\left(E_{j}+V\right)=\varnothing, i \neq j ; i, j=1, \ldots, N$,

(ii) for each $i$ there exists $\mu_{i} \in \mathscr{B}(G)$ such that $E_{\mu_{i}}=E_{i}$ and $\hat{\mu}_{i}(\gamma)=0, \gamma \notin E_{i}+V$.

Let $\mu=\mu_{1}+\cdots+\mu_{N}$. It is clear that $E_{\mu}=E$, and $\mu^{n}=\mu_{1}^{n}+\cdots+\mu_{N}^{n}, n=1,2, \ldots$, so $\mu \in \mathscr{B}(G)$.

RemarK. We are unable to determine whether or not the hypothesis that the $E_{i}$ are uniformly separated is necessary or not. For example, we do not know whether $\left\{(x, y) \in T^{2}: x=0\right.$ or $\left.y=0\right\}$ is in $\mathscr{E}\left(T^{2}\right)$ or whether $Z \cup \alpha Z, \alpha$ irrational, is in $\mathscr{E}(R)$, although both are closed sets in their respective coset rings.

We come now, after a lemma, to the characterization of $\mathscr{B}(G)$ mentioned at the beginning of this section.

(6.21) Lemma. Let $G$ be a compact abelian group and $\mu \in M(G)$. Let $E \in \mathscr{R}(\Gamma)$ and $\eta \in M(G)$ such that $\hat{\eta}=\chi_{E}$. The following are equivalent:

(i) $\mu^{n} * \eta=(\mu * \eta)^{n} \rightarrow 0$, as $n \rightarrow \infty$, in the weak-* topology.

(ii) $\mu^{n} * \eta \rightarrow 0$ in the strong convolution-operator topology on $L^{1}(G)$.

(iii) For each $f \in C(G)$ such that $\hat{f}(\gamma)=0$ if $-\gamma \notin E$, we have

$$
\int_{G} f d \mu^{n}=\int_{G} \cdots \int_{G} f\left(x_{1}+\cdots+x_{n}\right) d \mu\left(x_{1}\right) \cdots d \mu\left(x_{n}\right) \rightarrow 0 \text { as } n \rightarrow \infty .
$$

(iv) $\int_{G} f d \mu^{n} \rightarrow 0$ as $n \rightarrow \infty$ for every $f \in C(G)$ that is the uniform limit of trigonometric polynomials of the form $p(x)=\sum_{-\gamma \in E} c_{\gamma}(x, \gamma)$.

Proof. If (i) holds, by the Uniform Boundedness Principle there is a positive number $M$ such that $\left\|\mu^{n} * \eta\right\|<M, n=1,2, \ldots$ Let $f \in L^{1}(G)$, and for any $\varepsilon>0$ choose a trigonometric polynomial $p(x)=\sum_{1}^{m} c_{i}\left(x, \gamma_{i}\right)$ such that $\|f-p\|_{1}<\varepsilon / 2 M$. Applying (i) to the characters $\gamma \in \Gamma$ we see that $\left(\mu^{n} * \eta\right)^{\wedge}(\gamma) \rightarrow 0, \gamma \in \Gamma$. Thus for $n$ sufficiently large we have

$$
\begin{aligned}
\left\|p * \mu^{n} * \eta\right\|_{1} & =\left\|\sum_{i=1}^{m} c_{i}\left(\mu^{n} * \eta\right)^{\wedge}\left(\gamma_{i}\right)\left(x, \gamma_{i}\right)\right\|_{1} \\
& \leqq \sum_{i=1}^{m}\left|c_{i}\right|\left|\left(\mu^{n} * \eta\right)^{\wedge}\left(\gamma_{i}\right)\right|<\frac{\varepsilon}{2}
\end{aligned}
$$

giving $\left\|f * \mu^{n} * \eta\right\|_{1}<\varepsilon M / 2 M+\varepsilon / 2=\varepsilon$, and (ii) follows. Given (ii), we may again conclude that $\left(\mu^{n} * \eta\right)^{\wedge}(\gamma) \rightarrow 0, \gamma \in \Gamma$, and a similar argument, this time requiring $\|f-p\|_{G}<\varepsilon / 2 M$, yields (i).

It is a well-known fact that the functions $f$ which appear in (iii) are the same as those which appear in (iv) (see [21, 38D, p. 155]). Let $f \in C(G)$, and let $g(x)=f(-x)$, $x \in G$. Then

$$
\begin{aligned}
\int_{G} f d \mu^{n} * \eta & =\int_{G} g(-x) d \mu^{n} * \eta(x)=\int_{G} \int_{G} g(-x-y) d \eta(y) d \mu^{n}(x) \\
& =\int_{G} g * \eta(-x) d \mu^{n}(x) .
\end{aligned}
$$


If $h(x)=g * \eta(-x), x \in G$, then

$$
\hat{h}(\gamma)=(g * \eta)^{\wedge}(-\gamma)=\hat{g}(-\gamma) \hat{\eta}(-\gamma)=\hat{f}(\gamma) \hat{\eta}(-\gamma), \quad \gamma \in \Gamma .
$$

If (i) holds and $f$ is as in (iii), then (9) implies that $h=f$; whence (iii) follows by (8). Assume (iii) and let $f \in C(G)$. Then $h$ satisfies the hypothesis of (iii), and (8) gives (i).

(6.22) THEOREM. Let $G$ be a compact abelian group and $\mu \in M(G)$. Then $\mu \in \mathscr{B}(G)$ if and only if $\mu$ satisfies the following conditions:

(i) $\|\hat{\mu}\|_{\Gamma} \leqq 1, E_{\mu} \in \mathscr{R}(\Gamma)$, and $\hat{\mu} \mid E_{\mu}$ is piecewise affine.

(ii) $\mu$ satisfies one (and hence all) of the conditions in (6.21) with $E=\Gamma \mid E_{\mu}$ and $\eta$ as in (6.21).

Proof. Suppose $\mu \in \mathscr{B}(G)$. We have shown that (i) holds. Let $\hat{\eta}=\chi_{E}$. Then $\mu * \eta \in \mathscr{B}(G)$ and $\left[(\mu * \eta)^{\wedge}(\gamma)\right]^{n} \rightarrow 0, \gamma \in \Gamma$, so the only possible weak-* cluster point of the sequence $\left((\mu * \eta)^{n}\right)_{n=1}^{\infty}$ is 0 . It follows that (6.21.i) holds.

Now assume (i) and (6.21.i) holds, and let $\nu=\mu-\mu * \eta$. Then $\mu^{n}=(\mu * \eta)^{n}+\nu^{n}$, $n=1,2, \ldots$ We remarked in the proof of (6.21) that $\mu * \eta \in \mathscr{B}(G)$, while $\nu$ has the form (7) and hence is in $\mathscr{B}(G)$ as shown in (6.7).

(6.23) CoROLlaRY. Let $G$ be locally compact abelian and $\mu \in M(G)$. Then $\mu \in \mathscr{B}(G)$ if and only if:

(i) $\|\hat{\mu}\|_{\Gamma} \leqq 1$ and $E_{\mu}$ and $\hat{\mu} \mid E_{\mu}$ are as in (6.2).

(ii) $\mu$ satisfies (6.21.iv) with $E=\Gamma \mid E_{\mu}$.

(6.24) EXAMPLE. On every nondiscrete locally compact abelian group $G$ there is a measure $\mu \in M(G)$ such that $\|\hat{\mu}\|_{\Gamma}<1$ and $\mu \notin \mathscr{B}(G)$.

Proof. In order to show that $M(G)$ is asymmetric Williamson has constructed a measure $\nu \in M(G)$ such that $-1 \leqq \hat{\nu}(\gamma) \leqq 1, \gamma \in \Gamma$, while $\hat{v}(M)= \pm i$, say $+i$, for some $M \in \mathscr{M}_{M(G)}$ [26, Theorem 5.3.4, p. 107]. Let $P$ be a complex polynomial satisfying $P(0)=0, P(i)=2$, and $|P(x)| \leqq \alpha<1$ if $-1 \leqq x \leqq 1$. Let $\mu=P(v)$. Then $\|\hat{\mu}\|_{\Gamma} \leqq \alpha$, while $\left\|\mu^{n}\right\| \geqq\left|\hat{\mu}(M)^{n}\right|=2^{n}, n=1,2, \ldots$ Note that $\nu \in M_{c}(G)$ so $\mu \in M_{c}(G)$.

The lifting problem we referred to in the introduction to this section is the following one: Given $H$ a closed subgroup of $G$ and $\mu \in \mathscr{B}(G / H)$, does there exist $\nu \in \mathscr{B}(G)$ such that $\nu$ maps to $\mu$ in the usual way, i.e., such that $\hat{v} \mid \Lambda=\hat{\mu}, \Lambda$ the annihilator of $H$ in $\Gamma$ ? If such a $\nu$ exists we shall say that $\mu$ can be lifted to $\mathscr{B}(G)$. Of course, if $K \subset H \subset G, K$ and $H$ closed subgroups of $G$, and $\mu \in \mathscr{B}(G / H)$ can be lifted to $\mathscr{B}(G)$, then $\mu$ can be lifted to $\mathscr{B}(G / K)$. For the remainder of this section $\Lambda$ will denote the annihilator of the closed subgroup $H$ of $G$.

(6.25) LemMA. Let $G=H \times K$. Then any $\mu \in \mathscr{B}(G / H)=\mathscr{B}(K)$ can be lifted to $\mathscr{B}(G)$. Moreover, if $\Lambda$ is $a G_{\delta}$ in $\Gamma$ (i.e., the annihilator $\Delta$ of $K$ is metrizable), then we may choose $\nu$ so that $E_{v}=E_{\mu}$.

Proof. The first statement is obvious since $\mu$ itself may be considered as a member of $\mathscr{B}(G)$. If $\Delta$ is metrizable we can choose $\sigma \in M(H)$ such that $\|\sigma\|=1$ and 
$E_{\sigma}=\{0\}$. Let $\nu=\sigma \times \mu$. Then $\nu^{n}=\sigma^{n} \times \mu^{n}, n=1,2, \ldots$, so $\nu \in \mathscr{B}(G)$; and $\hat{\nu}(\gamma)=$ $\hat{\sigma}\left(\gamma_{1}\right) \hat{\mu}\left(\gamma_{2}\right), \gamma=\left(\gamma_{1}, \gamma_{2}\right) \in \Gamma=\Delta \times \Lambda$, which gives $\hat{\nu}(\gamma)=\hat{\mu}(\gamma)$ if $\gamma \in \Lambda$ and $|\hat{\nu}(\gamma)|<1$ if $\gamma \notin \Lambda$.

(6.26) LemMA. Let $H$ be compact. Then any $\mu \in \mathscr{B}(G / H)$ can be lifted to $\mathscr{B}(G)$ so that $E_{v}=E_{\mu}$.

Proof. This is obvious: simply choose $\nu \in M(G)$ such that $\hat{\nu}=\hat{\mu}$ on $\Lambda$ and $\hat{v}=0$ outside $\Lambda$.

(6.27) Lemma. If $G / H$ is compact, then every $f \in \mathscr{B}\left(L^{1}(G / H)\right)$ can be lifted to $\mathscr{B}\left(L^{1}(G)\right)$. If $\Gamma$ is metrizable, then we may require $E_{v}=E_{f}$.

Proof. Let $f \in \mathscr{B}\left(L^{1}(G / H)\right)$ and choose $g \in L^{1}(G)$ such that $g^{H}=f$, a.e. Since $\Lambda$ is discrete we may choose:

(i) an open set $U$ in $\Gamma$ such that $E_{f} \subset E_{g} \subset U$ and $\bar{U}$ is compact;

(ii) a symmetric neighborhood $W$ of 0 in $\Gamma$ such that $\tau+W \subset U, \tau \in U \cap \Lambda$ and $\Lambda \cap(W+W)=\{0\}$; and

(iii) a neighborhood $V$ of 0 in $\Gamma$ such that $(U+V) \cap \Lambda=U \cap \Lambda$.

Let $\alpha \in L^{1}(G)$ such that $\hat{\alpha} \equiv 1$ on $U, \hat{\alpha} \equiv 0$ off $U+V, 0 \leqq \hat{\alpha} \leqq 1$. Let $\beta \in L^{1}(G)$ with $\hat{\beta}(0)=1=\|\beta\|_{1}$ and $\hat{\beta} \equiv 0$ off $W$. If $\Gamma$ is metrizable we may also require $E_{\beta}=\{0\}$. Let

Then

$$
k(x)=g(x)-g * \alpha(x)+\sum_{\tau \in \Lambda \cap U} \hat{f}(\tau)(x, \tau) \beta(x), \text { a.e. }
$$

$$
\hat{k}(\gamma)^{n}=\hat{g}(\gamma)^{n}(1-\hat{\alpha}(\gamma))^{n}+\sum_{\tau \in \Lambda \cap U} \hat{f}(\tau)^{n} \hat{\beta}(\gamma-\tau)^{n}, \quad n=1,2, \ldots, \gamma \in \Gamma .
$$

Thus $\hat{k} \equiv \hat{f}$ on $\Lambda$, and if $\Gamma$ is metrizable $E_{k}=E_{f}$. Since $\left\|(g-g * \alpha)^{\wedge}\right\|_{\Gamma}<1$, we have $k \in \mathscr{B}\left(L^{1}(G)\right)$ as in the proof of (2.2).

(6.28) THEOREM. Let $G$ be compactly generated. Then every $f \in \mathscr{B}\left(L^{1}(G / H)\right)$ can be lifted to $\mathscr{B}\left(L^{1}(G)\right)$ so that $E_{v}=E_{f}$.

Proof. Fix $f \in \mathscr{B}\left(L^{1}(G / H)\right)$. Suppose first that $G=R^{p} \times Z^{q}$ and $\Gamma=R^{p} \times T^{q}$ for some nonnegative $p$ and $q$. We may write $\Lambda=R^{r} \times Z^{s} \times T^{t} \times F$ for some $0 \leqq r+s \leqq p$, $0 \leqq t \leqq q$, and $F$ a finite group, in such a way that $\Gamma$ may be written as $\Gamma=R^{p-r-s}$ $\times R^{r} \times R^{s} \times T^{t} \times T^{q-t}$ with $Z^{s} \subset R^{s}$ and $F \subset T^{q-t}$. In particular, $\Gamma=\Delta_{1} \times \Delta_{2}, \Delta_{1}$ and $\Delta_{2}$ being closed subgroups of $\Gamma$, and there exists a discrete subgroup $\Delta_{3}$ of $\Delta_{2}$ such that $\Lambda=\Delta_{1} \times \Delta_{3}$. If $\Delta_{3}=\{0\}$, our theorem follows from (6.25). If not, we argue exactly as in (6.27) on $\Delta_{2}$ by choosing $U \subset \Delta_{2}$ so that $E_{g} \subset \Delta_{1} \times U$. We arrive at the definition

$$
\begin{aligned}
k(x)=k\left(x_{1}, x_{2}\right)= & g\left(x_{1}, x_{2}\right)-g\left(x_{1}, x_{2}\right) *\left(\alpha\left(x_{2}\right) d \lambda_{2}\right) \\
& +\sum_{\tau \in \Delta_{3} \cap U} f^{\tau}\left(x_{1}\right)\left(x_{2}, \tau\right) \beta\left(x_{2}\right), \text { a.e., }
\end{aligned}
$$

where the meaning of $\lambda_{2}$ is clear and $f^{\tau} \in L^{1}(K)$ ( $K$ the annihilator of $\Delta_{1}$ in $G$ ) is 
chosen so that $\left(f^{\tau}\right)^{\wedge}\left(\gamma_{1}\right)=\hat{f}\left(\gamma_{1}, \tau\right), \gamma_{1} \in \Delta_{1}$. The proof is completed as in (6.27), noting that each $f^{\tau} \in \mathscr{B}(K)$ since $f \in \mathscr{B}(G / H)$ and $H \subset K$.

In the general case we have by the structure theorem for compactly generated abelian groups [15, Theorem (9.8), pp. 90-92] that $G=R^{p} \times Z^{q} \times F, F$ a compact group, so $\Gamma=R^{p} \times T^{q} \times D, D$ being discrete. Let $g \in L^{1}(G)$ such that $g^{H}=f$ (a.e.) and choose $\gamma_{1}, \ldots, \gamma_{n} \in D$ so that $E_{g} \subset R^{p} \times T^{q} \times\left\{\gamma_{1}, \ldots, \gamma_{n}\right\}$. On each of the cosets $\Delta_{i}=R^{p} \times T^{q} \times\left\{\gamma_{i}\right\}$ we may, by translating and applying the previous case, choose a $k_{i} \in \mathscr{B}\left(L^{1}\left(R^{p} \times Z^{q}\right)\right)$ such that $\hat{k}_{i}\left(\gamma-\gamma_{i}\right)=\hat{f}(\gamma), \gamma \in \Lambda \cap \Delta_{i}$, and $\gamma_{i}+E_{k_{i}}$ $=E_{f} \cap \Delta_{i}$. Let $k \in L^{1}(G)$ be the function whose transform is (cf. (6.26))

$$
\begin{aligned}
& \hat{k}(\gamma)=\hat{k}_{i}\left(\gamma-\gamma_{i}\right) \quad \gamma \in \Delta_{i}, \quad i=1, \ldots, n \\
& =\hat{g}(\gamma) \quad \gamma \notin \Delta_{1} \cup \cdots \cup \Delta_{n}, \quad \gamma \in \Gamma \text {. }
\end{aligned}
$$

Then $d \nu=k d \lambda_{G}$ clearly satisfies all the requirements of our theorem.

7. Some differentiability conditions. We shall generalize the following theorem of Strang [31] to functions of several variables in order to obtain sufficient conditions for functions $f \in L^{1}(G), G$ noncompact, to be in $\mathscr{B}(G)$.

THEOREM. Let $f \in L^{1}(Z)$ such that $\hat{f} \in C^{2}(T),\|\hat{f}\|_{T}=1$, and $E_{f}=\left\{e^{i \theta_{1}}, \ldots, e^{i \theta_{n}}\right\}$, $-\pi \leqq \theta_{k} \leqq \pi$. Assume that for $k=1, \ldots, n$ there are constants $\alpha_{k}, \gamma_{k}$, and $m_{k}$, where $\alpha_{k} \in R, \operatorname{Re} \gamma_{k}>0$, and $m_{k}$ is an even natural number, such that $f\left(e^{i \theta}\right) \in C^{m_{k}}$ in a neighborhood of $\theta=\theta_{k}$ and

$$
\hat{f}\left(e^{i\left(\theta+\theta_{k}\right)}\right)=\hat{f}\left(e^{i \theta_{k}}\right) \exp \left[i \alpha_{k} \theta-\gamma_{k} \theta^{m_{k}}(1+o(1))\right] \text { as } \theta \rightarrow 0 .
$$

Then $f \in \mathscr{B}(Z)$.

Thomée [33] has shown that in case $\hat{f}$ is analytic the conditions (10) are also necessary; in fact if $\hat{f}$ is analytic and does not behave as in (10) near every point of $E_{f}$ then $\left\|f^{n}\right\|_{1} \geqq C n^{\alpha}$ for some $0<\alpha<1$. For some generalizations of these results see [13], [14], and [34], [29], [30].

To avoid confusion we shall write $f^{n}$ for the $n$th pointwise power of a function $f$ on $R^{r}$ and $f^{(n)}$ for the $n$th convolution power. Elements of $G=R^{r}$ will be denoted by $x$ and those of $\Gamma=R^{r}$ by $y ; \partial_{j}^{i}$ denotes $\partial^{i} / \partial y_{j}^{i}$.

(7.1) LEMMA. Let $f \in C^{p}(R)$ and $n \geqq p$. Then

$$
D^{p} f^{n}=\sum_{k=1}^{p} \sum a_{i_{1}, \ldots, i_{k}} n(n-1) \cdots(n-k+1) f^{n-k} D^{i_{1}} f \cdots D^{i_{k}} f,
$$

the second summation being taken over all choices of $i_{1}, \ldots, i_{k}$ such that $1 \leqq i_{1} \leqq \ldots$ $\leqq i_{k} \leqq p$ and $i_{1}+\cdots+i_{k}=p$. The coefficients $a_{i_{1}, \ldots, i_{k}}$ are independent of $f$ and $n$.

(7.2) LEMMA. Let $f \in C_{00}^{m} \cap C^{r+1}\left(R^{r}\right), m$ an even natural number. Suppose that $|f(y)|<1$ if $y \neq 0$ and in a neighborhood of 0

$$
f(y)=\exp \left(-p(y)+o\left(\|y\|^{m}\right)\right) \text { as } y \rightarrow 0
$$


where $p(y)$ is a homogeneous polynomial of degree $m$ such that $\operatorname{Re} p(y)>0$ if $y \neq 0$. Then there exists $C>0$ such that

(i) $\left|\left(f^{n}\right)^{\wedge}(x)\right| \leqq C n^{-r / m}, x \in R^{r}$.

(ii) $\left|\left(f^{n}\right)^{\wedge}(x)\right| \leqq C n^{1 / m}\left|x_{j}\right|^{-(r+1)}, j=1, \ldots, r ; x=\left(x_{1}, \ldots, x_{r}\right) \in R^{r}, x_{j} \neq 0$.

Proof. Since $f$ has compact support and satisfies (11) in a neighborhood of 0 there exists $\alpha>0$ such that $|f(y)| \leqq \exp \left(-\alpha\|y\|^{m}\right), y \in R^{r}$. Thus we have by a change of variables,

$$
\begin{aligned}
\left|\left(f^{n}\right)^{\wedge}(x)\right| & \leqq(2 \pi)^{-r / 2} \int_{R^{r}}|f(y)|^{n} d y \leqq(2 \pi)^{-r / 2} \int_{R^{r}} \exp \left(-n \alpha\|y\|^{m}\right) d y \\
& =\frac{1}{(2 \pi)^{r / 2} n^{r / m}} \int_{R^{r}} \exp \left(-\alpha\|y\|^{m}\right) d y,
\end{aligned}
$$

which gives (i).

Fix $j \leqq r$. Integrating by parts $r+1$ times we obtain (for $x_{j} \neq 0$ )

$$
\left(f^{n}\right)^{\wedge}(x)=(2 \pi)^{-r / 2} \int_{R^{r}} f(y)^{n} e^{-i x \cdot y} d y=\frac{i^{r+1}}{(2 \pi)^{r / 2} x_{j}^{r+1}} \int_{R^{r}} \partial_{j}^{r+1} f^{n}(y) e^{-i x \cdot y} d y .
$$

Assume $n \geqq r+1$ and apply (7.1) to the integrand above, giving

$$
\left|\left(f^{n}\right)^{\wedge}(x)\right| \leqq \frac{\text { Const }}{\left|x_{j}\right|^{r+1}} \sum n^{k} \int_{R^{r}}\left|f^{n-k} \partial_{j}^{i} 1 f \cdots \partial_{j}^{i} k f\right| d y,
$$

the summation being taken over all choices of $k$ and $i_{1}, \ldots, i_{k}$ as in Lemma (7.1). From (11) we see that $f(y)=1-p(y)+o\left(\|y\|^{m}\right)$ as $y \rightarrow 0$. Note that the polynomial $p(y)$ must have a term of the form $a_{j} y_{j}^{m}\left(a_{j} \neq 0\right)$. For otherwise every term of $p$ would have a variable factor other than $y_{j}$, and we would have

$$
p\left(0, \ldots, 0, y_{j}, 0, \ldots, 0\right)=0,
$$

contradicting the nature of $p(y)$. Thus $\partial_{j}^{i} p$ is a homogeneous polynomial of degree $m-i, i=1, \ldots, m$. By Taylor's formula we have $\left|\partial_{j}^{i} f(y)\right| \leqq$ Const $\|y\|^{m-i}$, $i=1, \ldots, m$. If $m \leqq r+1$, we also have $\partial_{j}^{i} f$ bounded, $m+1 \leqq i \leqq r+1$. If we apply these estimates to the integrals on the right-hand side of (12) we obtain, for any choice of $k$ and $i_{1}, \ldots, i_{k}$,

where

$$
\begin{aligned}
\int_{R^{r}}\left|f^{n-k} \partial_{j}^{i} f f \cdots \partial_{j}^{i} k f\right| d y & \leqq \text { Const } \int_{R^{r}} \exp \left(-n \alpha\|y\|^{m}\right)\|y\|^{\beta} d y \\
& =\text { Const } n^{-(r+\beta) / m} \int_{R^{r}} \exp \left(-\alpha\|y\|^{m}\right)\|y\|^{\beta} d y,
\end{aligned}
$$

$$
\begin{aligned}
\beta & =k m-r-1 & & \text { if } i_{k} \leqq m \\
& =h m-i_{1}-\cdots-i_{h} & & \text { if } i_{h} \leqq m<i_{h+1} \\
& =0 & & \text { if } m<i_{1} \\
& \geqq k m-r-1 . & &
\end{aligned}
$$

This gives

$$
\int_{R^{r}}\left|f^{n-k} \partial_{j^{i}}^{i} f \cdots \partial_{j}^{i} k f\right| d y \leqq \text { Const } n^{(1 / m)-k} \int_{R^{r}} \exp \left(-\alpha\|y\|^{m}\right)\|y\|^{\beta} d y
$$

for each $k$ and $i_{1}, \ldots, i_{k}$, which when substituted in (12) completes the proof of (ii). 
(7.3) Corollary. Let $f \in L^{1}\left(R^{r}\right)$ and suppose $\hat{f}$ satisfies the hypotheses of Lemma (7.2). Then $f \in \mathscr{B}\left(R^{r}\right)$.

Proof. By the inversion theorem and (7.2) we have

(i) $\left|f^{(n)}(x)\right| \leqq C n^{-r / m}, x \in R^{r}$.

(ii) $\left|x_{j}^{r+1} f^{(n)}(x)\right| \leqq C n^{1 / m}, j=1, \ldots, r ; x=\left(x_{1}, \ldots, x_{r}\right) \in R^{r}$.

Adding the inequalities (ii) yields

$$
\left|f^{(n)}(x)\right| \leqq \frac{C n^{1 / m}}{\left|x_{1}\right|^{r+1}+\cdots+\left|x_{r}\right|^{r+1}} \leqq \frac{C^{\prime} n^{1 / m}}{\|x\|^{r+1}}, \quad x \in R^{r} \backslash\{0\} .
$$

Let $A_{r}$ denote the volume of the unit ball in $R^{r}$, and note that for any $a>0$

$$
\int_{\|x\|>a}\|x\|^{-(r+1)} d x=(2 \pi)^{r-1} \int_{a}^{\infty} \rho^{-2} d \rho=(2 \pi)^{r-1} / a .
$$

Thus by applying (i) and (13) we obtain

$$
\begin{aligned}
\left\|f^{(n)}\right\|_{1} & =(2 \pi)^{-r / 2} \int_{R^{r}}\left|f^{(n)}(x)\right| d x=(2 \pi)^{-r / 2}\left[\int_{\|x\| \leqq n^{1 / m}}+\int_{\|x\|>n^{1 / m}}\right] \\
& \leqq C_{1} n^{-r / m} A_{r} n^{r / m}+C_{2} n^{1 / m} n^{-1 / m}=C_{1} A_{r}+C_{2}, \quad n=1,2, \ldots
\end{aligned}
$$

(7.4) LeMma. Let $p(y)$ be a homogeneous polynomial of degree $m$ on $R^{t}, m$ an even positive integer, such that for some $s<t \operatorname{Re} p\left(y_{1}, \ldots, y_{s}, 0, \ldots, 0\right)>0$ unless $y_{1}=\cdots=y_{s}=0$. Then there exist positive numbers $a_{s+1}, \ldots, a_{t}$ such that $\operatorname{Re} p(y)$ $+a_{s+1} y_{s+1}^{m}+\cdots+a_{t} y_{t}^{m}>0$ if $y \neq 0$.

Proof. This follows from an elementary compactness argument, once it is noticed that it suffices to obtain the result for $y$ in the unit sphere of $R^{t}$.

(7.5) THEOREM. Let $f \in L^{1}\left(R^{r}\right)$, let $m_{1}, \ldots, m_{k}$ be even positive integers such that $m_{1}<m_{2}<\cdots<m_{k}$, and let $\varnothing=S_{0} \subset S_{1} \subset S_{2} \subset \ldots \subset S_{k}=\{1, \ldots, r\}$. Suppose $\hat{f} \in C^{m_{k}}$ $\cap C^{r+1}\left(R^{r}\right),|\hat{f}(y)|<1$ if $y \neq 0$, and in some neighborhood $U$ of 0 we have

$$
\hat{f}(y)=c \exp \left[\text { ity }-p_{m_{1}}(y)-p_{m_{1}+1}(y)-\cdots-p_{m_{k}}(y)+o\left(\|y\|^{m_{k}}\right)\right]
$$

as $y \rightarrow 0$, where $|c|=1, t \in R$, and $p_{m_{1}}, \ldots, p_{m_{k}}$ are homogeneous polynomials satisfying the following conditions:

(i) $\operatorname{deg} p_{i}=i, i=m_{1}, \ldots, m_{k}$.

(ii) $m_{j} \leqq i<m_{j+1}$ implies that $p_{i}$ involves only the variables $y_{h}$ with $h \in S_{j}, j=1$, ...,k-1.

(iii) $\operatorname{Re} p_{m_{j}}(y)>0$ if $y \neq 0$ and $y_{h}=0$ for all $h \in S_{j-1}, j=1, \ldots, k$. Then $f \in \mathscr{B}\left(R^{r}\right)$.

Proof. We may clearly assume $c=1$ and $t=0$ in (14). For each $j=1, \ldots, k$ choose positive numbers $a_{h}^{(j)}, h \in S_{j-1}$, such that

$$
\operatorname{Re} p_{m_{j}}(y)+\sum_{h \in S_{j-1}} a_{h}^{(j)} y_{h}^{m_{j}}>0
$$


unless $y_{h}=0$ for all $h \in S_{j}$. The $a_{h}^{(j)}$ may be chosen by (ii), (iii) and Lemma (7.4). For $y \in U$,

$$
\begin{gathered}
\hat{f}(y)=\prod_{j=1}^{k} \exp \left[-\left(p_{m,}(y)+\sum_{n \in S_{j-1}} a_{h}^{(j)} y_{h}^{m_{j}}\right)-p_{m_{j}+1}(y)-\cdots-p_{m_{j+1}-1}(y)\right. \\
\left.+\sum_{h \in S_{j}} a_{h}^{(j+1)} y_{h}^{m_{j+1}}\right] \\
\cdot \exp \left[-\left(p_{m_{k}}(y)+\sum_{h \in S_{k-1}} a_{h}^{(k)} y_{h}^{m_{k}}\right)+o\left(\|y\|^{\left.m_{k}\right)}\right] .\right.
\end{gathered}
$$

By (i) and (ii) each of the factors $f_{j}, j=1, \ldots, k$, in this product is of the form (11) when considered as a function on $U_{j}=U \cap G_{j}$, where $G_{j}=\left\{y \in R^{r}: y_{h}=0\right.$ if $\left.h \notin S_{j}\right\}$, $j=1, \ldots, k$. Let $\phi_{j} \in C_{00}^{\infty}\left(G_{j}\right)$ such that $0 \leqq \phi_{j} \leqq 1, \phi_{j} \equiv 1$ on a neighborhood of 0 and $\phi_{j} \equiv 0$ off $U_{j}, j=1, \ldots, k$. By (7.3) the functions $g_{j}=f_{j} \phi_{j}$ are transforms on $G_{j}$ of $L^{1}$-functions with bounded convolution powers. If we now consider the $g_{j}$ as functions on $R^{r}$ which depend only on the coordinates $y_{h}, h \in S_{j}$, they become Fourier-Stieltjes transforms of measures $\mu_{j} \in \mathscr{B}\left(R^{r}\right), j=1, \ldots, k$. Let $\mu=\mu_{1} * \cdots$ $* \mu_{k}$. Then $\mu \in L^{1}\left(R^{r}\right)$ since $\mu_{k} \in L^{1}\left(R^{r}\right), \hat{\mu} \equiv \hat{f}$ on a neighborhood of 0 , and $\mu \in \mathscr{B}\left(R^{r}\right)$. By the Localization Theorem (3.4), $f \in \mathscr{B}\left(R^{r}\right)$.

(7.6) Corollary. Let $f \in L^{1}\left(R^{r}\right)$ such that $\|\hat{f}\|_{R^{r}}=1$ and $E_{f}=\left\{y_{1}, \ldots, y_{N}\right\}$. Suppose that for each $v=1,2, \ldots, N$ there is a neighborhood $U_{v}$ of $y_{v}$ such that $\hat{f}\left(y_{v}+y\right)$ has the form (14) on $U_{v}$ and that $\hat{f} \in C^{m(v)} \cap C^{r+1}$ on $U_{v}$, where $m(v)$ is the corresponding value of $m_{k}$ in (7.5). Then $f \in \mathscr{B}\left(R^{r}\right)$.

Proof. We may assume that the $U_{v}$ are pairwise disjoint and that $U_{v}=y_{v}+V$ for some neighborhood $V$ of $0, \nu=1, \ldots, N$. Choose $\phi \in C_{00}^{\infty}\left(R^{r}\right)$ such that $0 \leqq \phi \leqq 1$, $\phi \equiv 1$ on a neighborhood of 0 and $\phi \equiv 0$ off $V$. Then the functions $g_{v} \in L^{1}\left(R^{r}\right)$ such that $\hat{g}_{v}(y)=\phi(y) \hat{f}\left(y_{v}+y\right)$ satisfy the hypotheses of our theorem, so $g_{v} \in \mathscr{B}\left(R^{r}\right)$, $\nu=1, \ldots, N$. Let $g(x)=\sum_{1}^{N} e^{i x \cdot y_{v}} g_{v}(x)$ (a.e.). Then $g \in \mathscr{B}\left(R^{r}\right), \hat{g} \equiv \hat{f}$ on a neighborhood of $E_{f}$, and $|\hat{g}(y)|<1, y \notin E_{f}$. By (3.4), $f \in \mathscr{B}\left(R^{r}\right)$.

(7.7) Lemma. Let $H$ be a discrete group with character group $\Lambda$. Let $p$ be a positive integer and let $F=\left\{\left(\xi_{1}, \ldots, \xi_{p}\right) \in T^{p}: \xi_{i}=1\right.$ for some $\left.i \leqq p\right\}$. Suppose $f$ is a function defined on $T^{p} \times \Lambda$ which vanishes on a neighborhood $U$ of $F \times \Lambda$. Let $g$ be defined on $R^{p} \times \Lambda$ by

$$
\begin{aligned}
g\left(x_{1}, \ldots, x_{p}, \gamma\right) & =f\left(e^{i x_{1}}, \ldots, e^{i x_{p}}, \gamma\right) & & \text { if } 0 \leqq x_{i} \leqq 2 \pi, \quad i=1, \ldots, p \\
& =0 & & \text { otherwise. }
\end{aligned}
$$

Then there exists $u \in L^{1}\left(Z^{p} \times H\right)$ with $\hat{u}=f$ if and only if there exists $v \in L^{1}\left(R^{p} \times H\right)$ with $\hat{v}=g$. Moreover, there exist constants $c_{1}$ and $c_{2}$ depending only on $U$ such that whenever $u$ and $v$ exist for some $f$ as above we have $\|u\|_{1} \leqq c_{1}\|v\|_{1} \leqq c_{2}\|u\|_{1}$.

Proof. The case $p=1$ and $H=\{0\}$ is [26, Theorem 2.7.6, pp. 56-57]. The proof of our lemma is analogous, and we shall omit the details. 
(7.8) Corollary. Let $p$ and $q$ be positive integers and $G=R^{p} \times Z^{q}$. Let $f \in L^{1}(G)$, and suppose $\hat{f}\left(x_{1}, \ldots, x_{p}, e^{i \theta_{1}}, \ldots, e^{i \theta_{q}}\right)$ satisfies the hypotheses of $(7.6)$ on $\Gamma=R^{p} \times T^{q}$. Then $f \in \mathscr{B}(G)$.

Proof. The localization property allows us to consider only transforms $g$ on $\Gamma$ with small supports $S$ which, after translating, may be assumed to satisfy $\delta<x_{i}$ $<2 \pi-\delta, i=1, \ldots, p, \quad\left(x_{1}, \ldots, x_{p}, e^{i \theta_{1}}, \ldots, e^{i \theta} q\right) \in S$, for some $\delta>0$. We can then apply (7.7) with $H=Z^{q}$ to consider $g$ as a function on $T^{p+q}$ and then apply it again with $H=\{0\}$ to consider $g$ as a function on $R^{p+q}$. (7.6) now gives us our result.

REMARK. Every locally compact abelian group $G$ is the direct limit of its compactly generated subgroups, and the form of the extension of the corollary above to compactly generated groups (cf. (6.28)) and then to arbitrary noncompact $G$ is clear (cf. [5], [35]). Of course, in light of (5.2) this result can be expected to have content only for groups $G$ which are not the direct limit of their compact subgroups (i.e., when $\Gamma$ is not totally disconnected).

8. Unbounded powers in $L^{1}(G)$ and nonabelian groups. We showed in (5.2) that if $G$ is compact and abelian then every $f \in L^{1}(G)$ with $\|\hat{f}\|_{\Gamma} \leqq 1$ is in $\mathscr{B}(G)$. In this section we prove the converse of this result for a certain class of locally compact groups, namely that every $G$ in this class for which the above condition on $L^{1}(G)$ is satisfied must be compact and abelian. We begin with an application of the results in $\$ 2$.

For a compact group $G$ and $1 \leqq p<\infty$, let $T$ denote the left regular representation of $L^{1}(G)$ on $L^{p}(G)$. Then it is well known that for all $f \in L^{1}(G) T_{f}$ is a compact operator, and if $p=1$ then $\left\|T_{f}\right\|=\|f\|_{1}$ (cf. [36, pp. 53-54]).

(8.1) TheOREM. Let $G$ be a compact nonabelian group and $f \in L^{p}(G), 1 \leqq p<\infty$. Then $f \in \mathscr{B}\left(L^{p}(G)\right)$ if and only if $\operatorname{sp}_{L^{p}(G)}(f) \subset D$ and each $\lambda \in \operatorname{sp}_{L^{p}(G)}(f)$ with $|\lambda|=1$ is a simple eigenvalue of $T_{f}$ on $L^{p}(G)$.

Proof. Note that for any $p$ the inequalities $\left\|f^{n}\right\|_{1} \leqq\left\|f^{n}\right\|_{p} \leqq\left\|f^{n-1}\right\|_{1}\|f\|_{p}, n=1$, $2, \ldots$, show that $f \in \mathscr{B}\left(L^{p}(G)\right)$ if and only if $f \in \mathscr{B}(G)$. The fact that $L^{p}(G) \subset L^{1}(G)$ implies firstly that $\operatorname{sp}_{L^{1}(G)}(f) \subset \operatorname{sp}_{L^{p}(G)}(f)$ and secondly that the opposite containment holds also, since any $0 \neq \lambda \in \operatorname{sp}_{L^{p}(G)}(f)$ is an eigenvalue of the compact operator $T_{f}$. Moreover, if $g \in L^{1}(G)$ is such that

$$
0=\left(\lambda \delta_{e}-f\right)^{n} * g=\lambda^{n} g+\sum_{k=1}^{n}\left(\begin{array}{l}
n \\
k
\end{array}\right)(-1)^{k} \lambda^{n-k} f^{k} * g
$$

and $\lambda \neq 0$, then $g \in L^{p}(G)$. Thus $\lambda \in \operatorname{sp}_{L^{1}(G)}(f)$ is a simple eigenvalue of $T_{f}$ on $L^{1}(G)$ if and only if it is a simple eigenvalue of $T_{f}$ on $L^{p}(G)$. By (2.6) our theorem holds when $p=1$; our two previous remarks show that it holds for every $p$.

(8.2) Definition. A locally compact group $G$ has the strong $L^{1}$-power property (s. $L^{1}$-p.p.) if every $f \in L^{1}(G)$ with $r(f) \leqq 1$ is in $\mathscr{B}(G)$.

(8.3) COROLlaRY. If $G$ is compact and has the s. $L^{1}-p . p$., then $G$ is abelian. 
Proof. Since $L^{2}(G)$ is the orthogonal direct sum of full matrix algebras which are not all one-dimensional unless $G$ is abelian (Peter-Weyl Theorem), this result follows easily from (8.1).

(8.4) Proposition. If $G$ has the s. $L^{1}$-p.p. so do any open subgroup and any factor group $G / H, H$ a compact normal subgroup.

(8.5) LEMMA. Let $G$ be an infinite abelian group with the discrete topology. Then $G$ does not have the s. $L^{1}-p . p .(c f .(6.24))$.

Proof. Suppose first that $G=Z$, and let $f \in L^{1}(Z)$ such that $\|\hat{f}\|_{\Gamma}=1$ and $E_{f}$ is an infinite proper subset of $T$. Then $f \notin \mathscr{B}(G)$ by (6.4). Now if $G$ is any group with an element of infinite order our result follows by (8.4).

On the other hand, suppose every element of $G$ has finite order. Then every character on $\Gamma$, given by an element of $G$, has finite range in $T$, and it follows that every piecewise-affine map of $\Gamma$ into $T$ must have finite range. Thus, to complete the proof of the lemma, we show that there exists $f \in L^{1}(G)$ such that $|\hat{f}| \equiv 1$ and $\hat{f}$ has infinite range, and apply (6.2).

We shall construct $g \in L^{1}(G)$ such that $0 \leqq \hat{g}(\gamma) \leqq 1, \gamma \in \Gamma$, and $\hat{g}$ attains infinitelymany values. We then set $f=e^{i g}$. To construct $g$, let $\left(H_{n}\right)_{n=1}^{\infty}$ be a strictly increasing sequence of finite subgroups of $G$ with $H_{1}=\{0\}$. Let $a_{n}$ be the order of $H_{n}$ and $\Lambda_{n}$ its annihilator in $\Gamma$, so that $\left(\Lambda_{n}\right)_{n=1}^{\infty}$ is a strictly decreasing sequence of compact open subgroups of $\Gamma$. If $g_{n}=a_{n}^{-1} \chi_{H_{n}}$, then $\hat{g}_{n}=\chi_{\Lambda_{n}}$ and $\left\|g_{n}\right\|_{1}=1$. Let $g=\sum_{1}^{\infty} 2^{-n} g_{n}$. Then the range of $\hat{g}$ is $\left\{\sum_{1}^{m} 2^{-n}: m=1,2, \ldots\right\}$.

(8.6) THEOREM. Let $G$ be a locally compact abelian group with the s. $L^{1}-p . p$. Then $G$ is compact.

Proof. $G$ contains an open subgroup $G_{0}$ of the form $R^{p} \times F$ for some nonnegative integer $p$ and compact group $F$. If $G$ is not compact, then either $p>0$ or $G / G_{0}$ is infinite. If $p>0$ then (8.4) would imply that $R^{p}$ has the s. $L^{1}$-p.p., which is absurd (6.5). And if $p=0$, so that $G_{0}$ is compact, then (8.4) would imply $G / G_{0}$ has the s. $L^{1}$-p.p., contradicting (8.5).

(8.7) Definition. Let $\mathscr{G}$ denote the class of all locally compact groups $G$ with the following structure: $G$ has an open subgroup $G_{0}$ of finite index whose commutator subgroup has compact closure.

REMARKS. (i) In particular $\mathscr{G}$ contains all compact groups and abelian groups.

(ii) A locally compact group $G$ with center $Z(G)$ is called a $Z$-group if $G / Z(G)$ is compact. Grosser and Moskowitz have shown [11, Corollary 1, p. 331] that the commutator subgroup of every $Z$-group has compact closure. Thus every $Z$-group is in $\mathscr{G}$.

(iii) C. C. Moore has recently shown that any locally compact group $G$ with the property that every irreducible unitary representation of $G$ is finite dimensional is an inverse limit of finite extensions of $Z$-groups, and Robertson [25] has shown that this implies $G \in \mathscr{G}$. For further relationships of this type see [12], [25]. 
(8.8) THEOREM. Let $G$ be a locally compact group and $H$ an open subgroup whose commutator subgroup has compact closure. If $G$ has the s. $L^{1}-p . p$. then $H$ must be compact and abelian. Thus if $G \in \mathscr{G}$ then $G$ has the s. $L^{1}-p . p$. if and only if it is compact and abelian.

Proof. Apply (8.4) and (8.6).

We conjecture that a locally compact group has the strong $L^{1}$-power property if and only if it is compact and abelian.

\section{BIBLIOGRAPHY}

1. A. Beurling, Sur les intégrales de Fourier absolument convergentes et leur application à une transformation fonctionelle, Nionde Skandanaviska Matematikerkongressen, Helsingfors, 1938, pp. 345-366.

2. A. Beurling and H. Helson, Fourier-Stieltjes transforms with bounded powers, Math. Scand. 1 (1953), 120-126. MR 15, 307.

3. F. F. Bonsall and B. J. Tomiuk, The semi-algebra generated by a compact linear operator, Proc. Edinburgh Math. Soc. (2) 14 (1964/65), 177-196. MR 32 \#1557.

4. P. Brenner, Power bounded matrices of Fourier-Stieltjes transforms, Math. Scand. 22 (1968), 115-129. MR 39 \#6022.

5. F. Bruhat, Distributions sur un groupe localement compact et applications à l'étude des représentations des groupes p-adiques, Bull. Soc. Math. France 89 (1961), 43-75. MR 25 \#4354.

6. P. J. Cohen, On a conjecture of Littlewood and idempotent measures, Amer. J. Math. 82 (1960), 191-212. MR 24 \#A3231.

7. - On homomorphisms of group algebras, Amer. J. Math. 82 (1960), 213-226. MR 24 \#A3232.

8. J. Dixmier, Les moyennes invariantes dans les semi-groupes et leurs applications, Acta Sci. Math. Szeged 12 (1950), pars A, 213-227. MR 12, 267.

9. N. Dunford and J. Schwartz, Linear operators. I: General theory, Pure and Appl. Math., vol. 7, Interscience, New York, 1958. MR 22 \#8302.

10. J. E. Gilbert, On projections of $L^{\infty}(G)$ onto translation-invariant subspaces, Proc. London Math. Soc. (3) 19 (1969), 69-88. MR 39 \#6019.

11. S. Grosser and M. Moskowitz, On central topological groups, Trans. Amer. Math. Soc. 127 (1967), 317-340. MR 35 \#292.

12. - Compactness conditions in topological groups. I, II, J. Reine Angew. Math. (to appear).

13. G. W. Hedstrom, Norms of powers of absolutely convergent Fourier series, Michigan Math. J. 13 (1966), 393-416. MR 34 \#3193.

14. - Norms of powers of absolutely convergent Fourier series in several variables, Michigan Math. J. 14 (1967), 493-495. MR 36 \#5599.

15. E. Hewitt and K. A. Ross, Abstract harmonic analysis. Vol. I: Structure of topological groups. Integration theory, group representations, Die Grundlehren der math. Wissenschaften, Band 115, Academic Press, New York and Springer-Verlag, Berlin and New York, 1963. MR 28 \#158.

16. E. Hewitt and H. Rubin, The maximum value of a Fourier-Stieltjes transform, Math. Scand. 3 (1955), 97-102. MR 17, 172.

17. E. Hille and R. S. Phillips, Functional analysis and semi-groups, rev. ed., Amer. Math. Soc. Colloq. Publ., vol. 31, Amer. Math. Soc., Providence, R. I., 1957. MR 19, 664.

18. T. Itô and I. Amemiya, A simple proof of the theorem of P. J. Cohen, Bull. Amer. Math. Soc. 70 (1964), 774-776. MR 29 \#4862. 
19. M. A. Kaashoek and T. T. West, Locally compact monothetic semi-algebras, Proc. London Math. Soc. (3) 18 (1968), 428-438. MR 37\# 3358.

20. T. Kato, Estimation of iterated matrices, with application to the von Neumann condition, Numer. Math. 2 (1960), 22-29. MR 22 \#711.

21. L. H. Loomis, An introduction to abstract harmonic analysis, The University Series in Higher Math., Van Nostrand, Princeton, N. J., 1953. MR 14, 883.

22. J. J. H. Miller, On power-bounded operators and operators satisfying a resolvent condition, Numer. Math. 10 (1967), 389-396. MR 36 \#3147.

23. R. D. Richtmyer and K. W. Morton, Difference methods for initial-value problems, 2nd ed., Interscience Tracts in Pure and Appl. Math., no. 4, Interscience, New York, 1967. MR 36 \#3515.

24. C. E. Rickart, General theory of Banach algebras, The University Series in Higher Math., Van Nostrand, Princeton, N. J., 1960. MR 22 \#5903.

25. L. C. Robertson, Note on the structure of Moore groups, Bull. Amer. Math. Soc. 75 (1969), 594-598.

26. W. Rudin, Fourier analysis on groups, Interscience Tracts in Pure and Appl. Math., no. 12, Interscience, New York, 1962. MR 27 \#2808.

27. B. M. Schreiber, Bounded iterates in Banach algebras, Thesis submitted to University of Washington, May 1968.

28. - On the coset ring and strong Ditkin sets, Pacific J. Math. 32 (1970), 805-812.

29. S. I. Serdjukova, A necessary and sufficient condition for stability in the uniform metric of systems of difference equations, Dokl. Akad. Nauk SSSR 173 (1967), 526-528=Soviet Math. Dokl. 8 (1967), 438-440. MR 36 \#4845.

30. - On stability in the uniform metric of systems of difference equations, Ž. Vyčisl. Mat. i Mat. Fiz. 7 (1967), 497-509. (Russian) MR 35 \#7023.

31. W. G. Strang, Polynomial approximation of Bernstein type, Trans. Amer. Math. Soc. 105 (1962), 525-535. MR 25 \#5318.

32. B. Sz.-Nagy, On uniformly bounded linear transformations in Hilbert space, Acta Univ. Szeged Sect. Sci. Math. 11 (1947), 152-157. MR 9, 191.

33. V. Thomée, Stability of difference schemes in the maximum-norm, J. Differential Equations 1 (1965), 273-292. MR 31 \#515.

34. V. Ja. Urm, Necessary and sufficient conditions for the stability of a system of difference equations, Dokl. Akad. Nauk SSSR 139 (1961), 40-43=Soviet Math. Dokl. 2 (1961), 873-876. MR 24 \#A337.

35. A. Wawrzyńczyk, On tempered distributions and Bochner-Schwartz theorem on arbitrary locally compact Abelian groups, Colloq. Math. 19 (1968), 305-318. MR 37 \#3355.

36. A. Weil, L'intégration dans les groupes topologiques et ses applications, 2nd ed., Actualités Sci. Indust., nos. 869, 1145, Hermann, Paris, 1951.

UNIVERSITY OF WASHINGTON, SEATtLe, Washington 98105

WAYNe STATE UNIVERSiTy, Detroit, Michigan 48202 